CERN-PPE/97-01

8 January 1997

\title{
A MEASUREMENT OF THE HOLOGRAPHIC MINIMUM-OBSERVABLE BEAM BRANCHING RATIO IN THE FERMILAB 15-FOOT BUBBLE CHAMBER
}

\author{
E-632 Collaboration \\ M. Aderholz ${ }^{11)}$, M.M. Aggarwal ${ }^{5)}$, H. Akbari ${ }^{17, a)}$, P.P. Allport ${ }^{12, b, c)}$, S.K. Badyal ${ }^{10)}$, \\ H.C. Ballagh ${ }^{1)}$, M. Barth ${ }^{3, d)}$, J.P. Baton ${ }^{15)}$, H.H. Bingham ${ }^{1, h)}$, H. Bjelkhagen ${ }^{6, e)}$, \\ E.B. Brucker ${ }^{16)}$, R.A. Burnstein ${ }^{9)}$, J.R. Campbell ${ }^{8)}$, R.J. Cence ${ }^{7)}$, T.K. Chatterjee $^{5)}$, \\ E.F. Clayton ${ }^{8)}$, G. Corrigan ${ }^{12)}$, C. Coutures ${ }^{15)}$, D. DeProspo ${ }^{13)}$, Devanand ${ }^{10)}$, \\ E.A. De Wolf ${ }^{3, f, g)}$, P.J.W. Faulkner ${ }^{2)}$, H. Foeth ${ }^{4)}$, W.B. Fretter ${ }^{1, h)}$, K. Geissler ${ }^{4}$, \\ V.K. Gupta ${ }^{10)}$, J. Hanlon ${ }^{6)}$, G.G. Harigel ${ }^{4)}$, F.A. Harris ${ }^{7)}$, J. Hawkins ${ }^{6, i)}$, M.A. Jabiol ${ }^{15)}$, \\ P. Jacques ${ }^{13)}$, G.T. Jones ${ }^{2)}$, M.D. Jones ${ }^{7)}$, T. Kafka ${ }^{17)}$, M. Kalelkar ${ }^{13)}$, P. Kasper ${ }^{15, j)}$, \\ J.M. Kohli5), E.L. Koller ${ }^{16)}$, R.J. Krawiec ${ }^{2)}$, M. Lauko ${ }^{13)}$, J.E. Lys ${ }^{1)}$, P. Marage ${ }^{3)}$, \\ R.H. Milburn ${ }^{17)}$, D.B. Miller ${ }^{8)}$, I.S. Mittra ${ }^{5)}$, M.M. Mobayyen ${ }^{8)}$, J. Moreels ${ }^{3)}$, \\ D.R.O. Morrison ${ }^{4)}$, G. Myatt ${ }^{12)}$, R. Naon ${ }^{9)}$, A. Napier ${ }^{17)}$, P. Naylor ${ }^{8, b)}$, M. Neveu ${ }^{15)}$, \\ D. Passmore ${ }^{17)}$, M.W. Peters ${ }^{7}$, V.Z. Peterson ${ }^{7}$, R. Plano ${ }^{13)}$, N.K. Rao ${ }^{10)}$, H.A. Rubin ${ }^{9)}$, \\ J. Sacton ${ }^{3)}$, S.S. Sambyal ${ }^{10)}$, N. Schmitz ${ }^{11)}$, J. Schneps ${ }^{17)}$, R.L. Sekulin ${ }^{14)}$, S. Sewell ${ }^{14)}$, \\ J.B. Singh ${ }^{5)}$, W. Smart ${ }^{6)}$, P. Stamer ${ }^{13, k)}$, K.E. Varvell ${ }^{2,1)}$, L. Verluyten ${ }^{3, \mathrm{~m})}$, L. Voyvodic ${ }^{6)}$, \\ H. Wachsmuth ${ }^{4)}$, S. Wainstein ${ }^{8, b)}$, W. Williams ${ }^{6)}$, S. Willocq ${ }^{3, n)}$ and G.P. Yost ${ }^{1)}$
}

\begin{abstract}
Holography has been used successfully in combination with conventional optics for the first time in a large cryogenic bubble chamber, the 15-Foot Bubble Chamber at Fermilab, during a physics run. The innovative system combined the reference beam with the object beam, illuminating a conical volume of $\sim 1.4 \mathrm{~m}^{3}$. Bubble tracks from neutrino interactions with a width of $\sim 120 \mu \mathrm{m}$ have been recorded with good contrast. The ratio of intensities of the object light to the reference light striking the film is called the Beam Branching Ratio. We obtained in our experiment an exceedingly small minimum-observable ratio of $(0.54 \pm 0.21) \times 10^{-7}$. The technology has the potential for a wide range of applications.
\end{abstract}

(Submitted to Nuclear Instruments and Methods in Physics Research) 
1) University of California, Berkeley, CA 94720, USA

2) University of Birmingham, Birmingham B15 2TT, United Kingdom

3) Inter-University Institute for High Energies (ULB-VUB), B-1050 Brussels, Belgium

4) CERN, CH-1211 Geneva 23, Switzerland

5) Punjab University, Chandigarh-160014, India

6) Fermilab, Batavia, IL 60510, USA

7) University of Hawaii, Honolulu, HI 96822, USA

8) Imperial College of Science and Technology, London SW7 2AZ, United Kingdom

9) Illinois Institute of Technology, Chicago, IL 60616, USA

10) University of Jammu, Jammu-180001, India

11) Max-Planck-Institut für Physik, D-80805 Munich, Germany

12) Department of Nuclear Physics, University of Oxford, Oxford OX1 3RH, United Kingdom

13) Rutgers University, New Brunswick, NJ 08903, USA

14) Rutherford Appleton Laboratory, Chilton, Didcot OX11 0QX, United Kingdom

15) Département de Physique des Particules Elémentaires, Centre d'Etudes Nucléaires, Saclay, F-91191 Gif-sur-Yvette, France

16) Stevens Institute of Technology, Hoboken, NJ 07030, USA

17) Tufts University, Medford, MA 02155, USA

a) Now at Charles River Associates Inc., Boston MR 02116, USA

b) Supported by a studentship from U.K. S.E.R.C.

c) Now at Cavendish Laboratory, University of Cambridge, Cambridge, United Kingdom.

d) Maître de Recherches, FNRS, Belgium.

e) Now at American Propylaea Corp., Birmingham, Mich. USA.

f) Research Director, NFWO, Belgium.

g) Also at Universitaire Instelling Antwerpen, B-2610 Wilrijk, Belgium.

h) Deceased.

i) Now at Argonne National Laboratory, Argonne, IL 60439, USA.

j) Now at Fermilab, Batavia, IL 60510, USA.

k) At Seton Hall University, S. Orange, NJ 07079, USA.

1) ANSTO, Private Mail Bag 1, Menai, NSW 2234, Australia.

m) Now with XEIKON, Mortsel, Belgium.

n) Now at SLAC, Stanford, CA 94309, USA. 
Since the invention of holography by Dennis Gabor in 1948 [1] the technique has found a multitude of scientific, industrial, and artistic applications, many of which were outlined in his Nobel Lecture in 1971 [2]. The invention of the laser as a source of coherent monochromatic light has allowed the field to expand dramatically owing to the availability of powerful, high-quality lasers with the choice of pulse duration between nanoseconds up to pulse-stretched versions of $\sim 100$ microseconds and wavelengths spanning the visible range. From a technical or physics point of view one can distinguish between the recording of static and of moving objects, and single and double (or even multiple) exposures of the object on the same film, the latter producing interference images. Most of the techniques require a large coherence length and mono-mode operation of the laser; others need the multi-mode performance of gaseous lasers, now also obtainable using solid-state lasers having many modes. Holograms of three-dimensional objects can be replayed and then observed from different directions, so that quantitative statements on linear displacements can be obtained, or that manufacturing defects can become apparent through the observation of minute deformations. A hologram records a volume in such a way that it removes the classical limitations of incoherent illumination in its ability to achieve simultaneously high resolution and large depth of focus. It is thus of importance to apply holographic techniques to the recording of three-dimensional particle interactions in a large bubble chamber. A few examples of holography, quoted from Ref. [2], will be reviewed briefly.

(i) "The size of droplets a few micrometers in diameter, which issue from a nozzle with a velocity of 2 Mach, can only be determined by holographic techniques using a Q-switched laser with a pulse duration of 20 to 30 ns". Single exposure of the hologram is sufficient and gives the desired result.

(ii) Another example of a fast moving object is "a bullet with a train of shock waves meeting another shock wave: holography produces an impressive interferometric image [3]. The fringes show the 'loci' at which the retardation of light is by integer wavelengths, relative to the quiet air, before the event. This comparison standard is obtained by a previous exposure. This is therefore a double exposure hologram".

(iii) "Holographic images taken of moving objects are washed out. But if double exposure is used, first with the object at rest, then in vibration, fringes will appear, indicating the lines where the displacement amounted to multiples of a half wavelength". Nice examples are reconstructed holograms from a moving loudspeaker membrane [4], or the vibrations of the body of a guitar.

(iv) "Small deformations of solid bodies show up in double exposures as Newton fringes. Waves, frozen in at different times, and which have never seen one another, will be revived simultaneously, and they interfere with one another, each fringe corresponding to a deformation of a half wavelength. For the study of larger deformations, two holograms can be taken of the same object, but with two wavelengths. This produces beats between the two-fringe system, with fringe spacing indicating the deformations". [5]

(v) Finally, we should mention the high 'storage capacity' of holograms measured in pixels distributed over a volume compared to conventional photography.

\subsection{Holography for bubble chambers}

As early as 1963 a proposal was made to apply holography to high-energy physics apparatus [6], then in 1968 some preliminary tests were done [7, 8], and the idea was revived shortly afterwards in a theoretical paper [9]. Some ten years later the classical Gabor tech- 
nique was applied to record, in small bubble chambers, strings of tiny bubbles produced during the passage of elementary particles. The feasibility of this high-resolution recording was demonstrated with the $120-\mathrm{cm}^{3}$ bubble chamber (Berne Infinitesimal Bubble Chamber - BIBC [10]). The first exposure of a 2000- $\mathrm{cm}^{3}$ chamber (Holographic Bubble Chamber - HOBC [11]) in a charged-particle beam at CERN for a physics experiment resulted in 40000 holograms, which were all replayed and analysed. The information recorded was the fringe pattern formed by the interference of a reference beam and the object beam (i.e. the light scattered by a bubble). The objects were gas bubbles with a refractive index of $\sim 1.0$, embedded in the liquid (Freon) with an index of $\sim 1.25$. For these two chambers the requirements on the energy of the laser beam were modest and allowed the use of Q-switched pulses of several tens of nanoseconds' duration.

However, none of the techniques described above could be applied without considerable modifications to the holography of bubble tracks in large bubble chambers. A $1.2-\mathrm{m}^{3}$ Tohoku-MIT bubble Chamber [12] was designed from the start as a holographic chamber. It used a two-beam technique where the reference beam did not pass through the liquid to be holographed. Illumination with a time-expanded Q-switched laser pulse $(\sim 1.5 \mathrm{~J})$ was required to avoid unwanted heating effects from high-peak power.

Another holographic bubble chamber (GINES [13]), under design at the Russian accelerator centre in Serpukhov, is similar in geometrical design to the Tohoku-MIT chamber and may use partially coherent multi-mode laser illumination [14].

\subsection{Holography of a very large bubble chamber}

For experiment E-632 the 15-Foot Bubble Chamber $\left(33 \mathrm{~m}^{3}\right)$ at Fermilab was filled with a $62 / 38 \mathrm{~mol} \% \mathrm{Ne} / \mathrm{H}_{2}$ mixture having a refractive index of only 1.088 . One of the aims of this experiment was to search for short-lived particle decays arising from neutrino interactions. This required the recording of tracks with higher resolution and over a much larger depth of focus than was possible with conventional optics. For the illumination of $\sim 100-\mu \mathrm{m}$ bubble tracks in two cubic metres at least $6 \mathrm{~J}$ of energy, the maximum available from the pulse-stretched laser, was needed. As had been shown during previous tests in the Big European Bubble Chamber (BEBC) at CERN [15, 16], a Q-switched pulse could not be used, since the instantaneous power at the relatively small entrance port for the light into the chamber liquid would be too high, producing immediate excessive heating of the liquid in the vicinity of the port, followed by boiling. A solution to this problem was to expand the laser pulse over a longer time with constant power, thus reducing the instantaneous power. The technically easiest solution, namely using standard freelasing pulses of $\sim 1$-ms duration, would avoid the unwanted heating effect, but had to be discarded because of bubble chamber dynamics. The growth of bubbles, their movement due to buoyancy forces, and the vibration of the entire installation during this 1-ms pulse duration caused the interference pattern between reference and object beam to be washed out. Double exposure of the same tracks is no solution for circumventing the smearing. The challenge was to look for an intermediate pulse duration to satisfy simultaneously the requirements for good quality holograms and negligible heating of the liquid. The reference and object beams had to be produced in a new way.

In the present paper we investigate the conditions that give the best-quality holograms for the recording of tiny vapour bubbles in the cryogenic liquid fill of the 15-Foot Bubble Chamber. A detailed description of the set-up, its operation, and the analysis of holograms is given in Ref. [17]. In Section 2 we describe briefly the experimental set-up (more details have been given in [18]); in Section 3 the conditions for data-taking; in Sec- 
tion 4 the selection of the holograms for the analysis and the method of measuring bubble sizes in various illumination systems (bright-field, dark-field holograms); and finally in Section 5 there is a critical discussion of the intensity ratio object-to-reference beam, henceforth referred to as the Beam Branching Ratio $(B B R)$. In our specific application the choice of this ratio is restricted by a variety of conditions. The $B B R$ needed to illuminate bubble tracks in a large volume with good contrast has not yet been studied in any of the other bubble chambers, and the knowledge of the 'minimum-observable $B B R$ ' in particular may become of interest for applications outside the field of high-energy physics.

\section{EXPERIMENTAL ARRANGEMENT}

The 15-Foot Bubble Chamber was built in the early seventies and is equipped with six optic ports at the top [19]. A wide-angle lens, surrounded by an annular flash tube, and camera are installed inside each of the six hemispherical windows (Maxwell fisheyes). Each light source illuminates the liquid through reflection from the inner surface of the chamber vessel, which is wall-papered with a retrodirective material (Scotchlite ${ }^{\circledR}$, 3M Company). The light is reflected back to the entrance pupil of its respective lens. If vapour bubbles are present they act as scattering objects and this part of the reflected light does not reach the lens, i.e. the film is not sensitized in that particular region. This type of bright-field illumination is described in detail elsewhere [20]. The diffraction-limited resolution of the standard lenses is $\sim 500 \mu \mathrm{m}$ in the mid plane of the chamber (beam plane), giving a depth of focus of $\sim \pm 1 \mathrm{~m}$, although more of the volume is generally used. Each camera sees $\sim 28 \mathrm{~m}^{3}$ out of the total chamber volume of $33 \mathrm{~m}^{3}$, but interactions are accepted for physics analysis only if their vertex is inside a restricted 'fiducial volume' of $\sim 12 \mathrm{~m}^{3}$. Simultaneous photographs are taken during the sensitive period of the bubble chamber and analysed later to reconstruct the bubble tracks in space. During the physics run one optic port was equipped with a special lens having a larger aperture. Its flash was fired earlier, when the bubbles were still small, so that a resolution of $\sim 200 \mu \mathrm{m}$ was obtained, but at the expense of a depth of field reduced to about $0.2 \mathrm{~m}$, corresponding to a volume of $\sim 0.5 \mathrm{~m}^{3}$. Information from this camera was used for special investigations, but not for stereo reconstruction of events.

The choices for the layout of holography for the 15-Foot Bubble Chamber were limited. The design we have chosen was imposed by the above geometry, the parameters outlined in Section 1, and by some additional requirements to be discussed later. Our layout has already been described in detail [18]. Therefore, we can limit ourselves here to a fairly brief description.

We decided to illuminate the chamber with the laser light from near the bottom of the chamber, following the suggestion of Charles Baltay (Columbia University), and selected an optic port opposite to the beam entrance to be the holographic camera [19] (Fig. 1). A simplified scheme of the set-up is shown in Fig. 2. It introduces the geometrical parameters $d_{\mathrm{i}}, d_{\mathrm{h}}, \alpha, \Theta_{\mathrm{i}}$ and $\Theta_{\mathrm{h}}$. The distance between the diverging lens and the bubble is denoted by $d_{\mathrm{i}}$; the distance between the film plane and the bubble by $d_{\mathrm{h}}$; the illumination angle $\Theta_{i}$ represents the angle between the holographic axis and the line connecting the bubble with the centre of the dispersing lens, $\Theta_{\mathrm{h}}$ denotes the angle between the holographic axis and the line connecting the bubble with the centre of the holographic film plane, and $\alpha$ is called the scattering angle, which is between the dispersing-lens-to-bubble direction and the bubble-to-film direction. The object light waves are scattered by the bubbles (which make up the particle tracks). The intensity of light at the film scattered at an 
angle $\alpha$ by a spherical bubble [21] is:

$$
I_{0}=\frac{I_{\mathrm{i}} r^{2} \cdot G(\alpha) \cdot \cos \Theta_{\mathrm{h}}}{d_{\mathrm{h}}^{2}},
$$

with $I_{\mathrm{i}}$ the light intensity hitting the bubble, $r$ the radius of the bubble and $\Theta_{\mathrm{h}}$ the tilt of the film plane with respect to the object beam; $G(\alpha)$ is called the scattering function. The function depends on the refractive index of the liquid surrounding the vapour bubble. $G(\alpha)$ can be calculated numerically and is displayed in Fig. 3 for bubbles in the $\mathrm{Ne} / \mathrm{H}_{2}$ mixture with a refractive index of 1.088 [21]. It is a rapidly decreasing function of the scattering angle $\alpha$. In order to holograph bubbles at larger angles, this rapidly decreasing function must be compensated by higher illumination intensities $I_{\mathrm{i}}$ to keep the light intensity $I_{0}$ from the bubbles at the film plane approximately constant. Therefore the laser light is spread out non-uniformly at the entrance of the bubble chamber. This requires a nonspherical diverging lens.

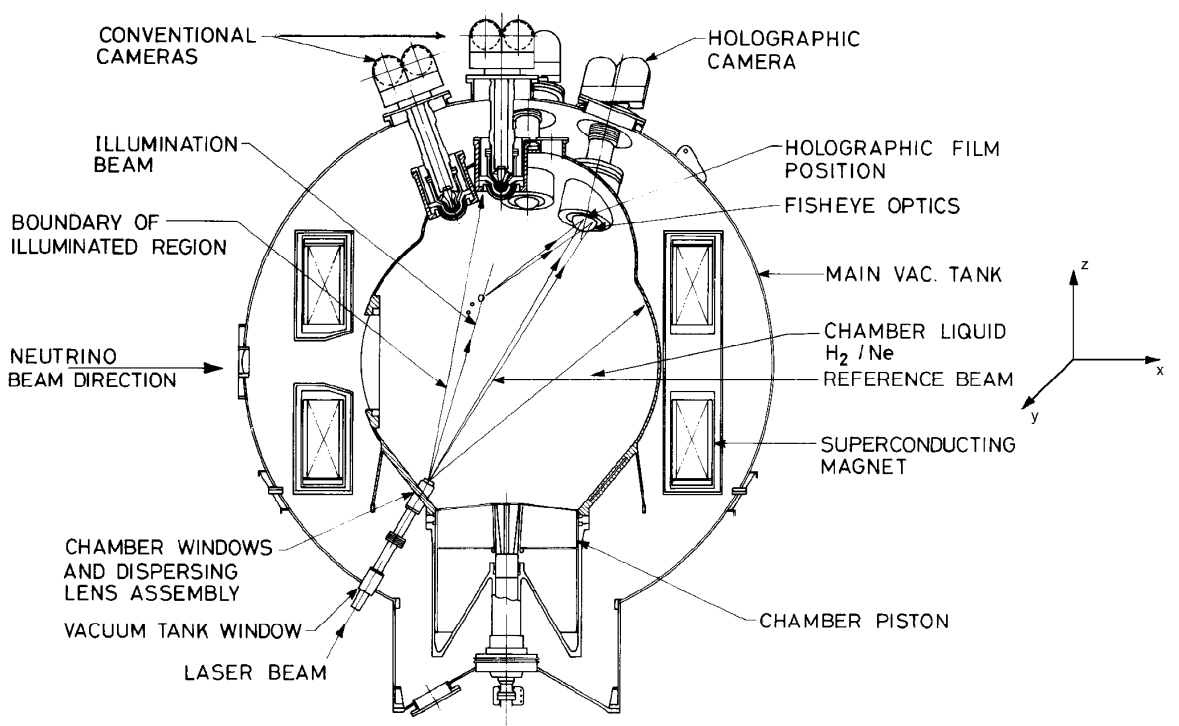

Figure 1: 15-Foot Bubble Chamber: side view.

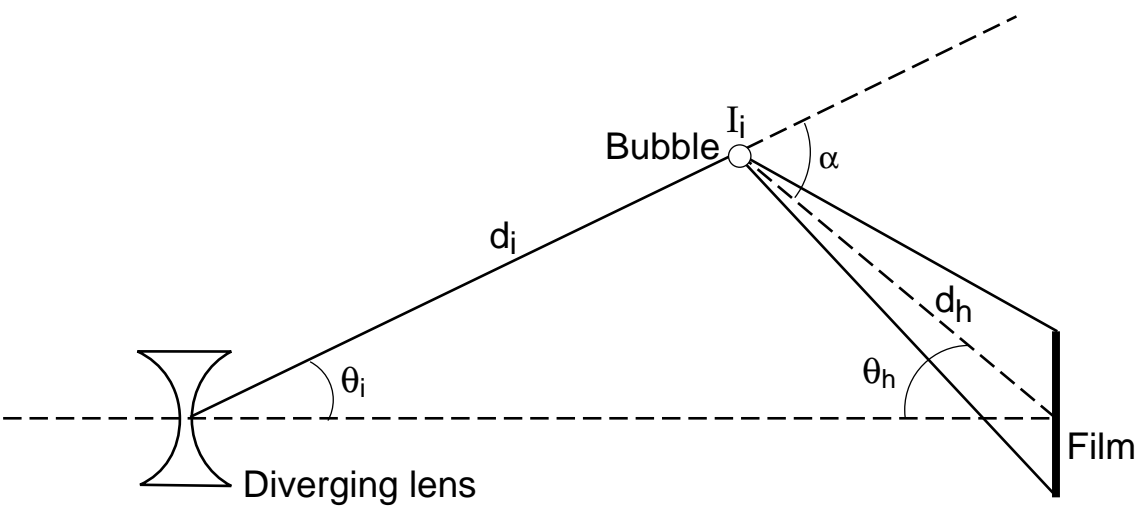

Figure 2: The holographic set-up (schematic). 


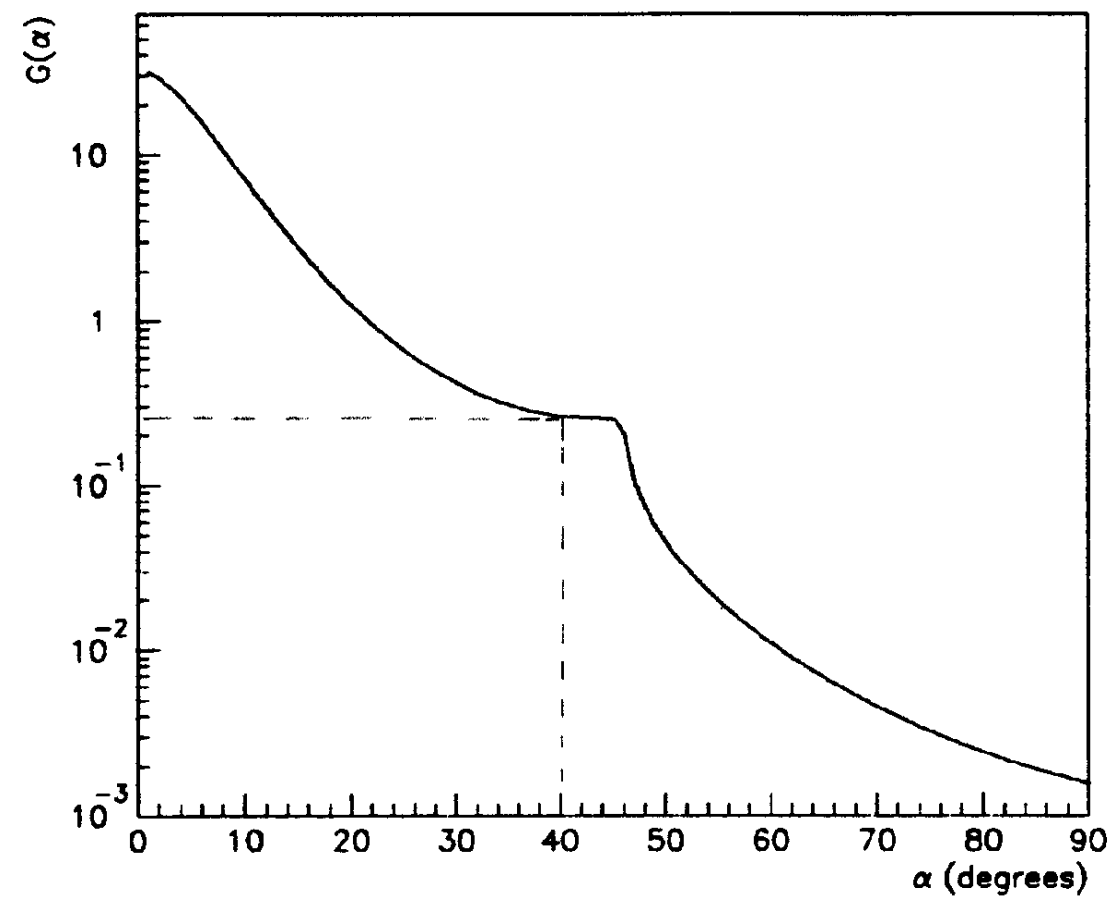

Figure 3: Light scattering function $G(\mathrm{a})$ for bubbles in a $62 / 38 \mathrm{~mol} \% \mathrm{Ne} / \mathrm{H}_{2}$ mixture as a function of the scattering angle $\alpha$. The dashed line gives the cut used during scanning.

The film is placed in the centre of the fish-eye window perpendicular to the reference beam direction within $1^{\circ}$. Furthermore, the light output distribution from the dispersing lens is described by a function $F\left(\Theta_{\mathrm{i}}\right)$, being the illumination intensity distribution per solid angle. Then the illumination light intensity hitting the bubble is given by $I_{\mathrm{i}}=F\left(\Theta_{i}\right) / d_{\mathrm{i}}^{2}$. The Beam Branching Ratio can thus be expressed as:

$$
B B R=\frac{I_{0}}{\left\langle I_{\mathrm{r}}\right\rangle}=\frac{F\left(\Theta_{\mathrm{i}}\right) r^{2} G(\alpha) \cos \Theta_{\mathrm{h}}}{\left\langle I_{\mathrm{r}}\right\rangle d_{\mathrm{i}}^{2} d_{\mathrm{h}}^{2}}
$$

with $\left\langle I_{\mathrm{r}}\right\rangle$ the reference beam intensity and the fringe visibility $V$, derived from theory, takes the form:

$$
V=2 \sqrt{B B R} \cos \Theta,
$$

with $\Theta$ the angle between the directions of polarization of the reference and object beams.

We define the $B B R^{\prime}$ as the actual Beam Branching Ratio needed, when noise light intensity $k$ times the reference beam is introduced, to get the same fringe modulation as was obtained without noise:

$$
B B R^{\prime}=B B R(1+k)^{2} ;
$$

thus the presence of noise requires a higher $B B R$ to obtain the same fringe modulation. In case of noise intensity equal to reference beam intensity, a four times higher $B B R$ is needed.

The visibility $V$ of the interference fringes is critically dependent on the design of the dispersing lens, as seen from these formulae. Indeed, the light output distribution 
$F\left(\Theta_{\mathrm{i}}\right)$ is entirely determined by the shape of the lens and the spatial distribution of the input laser beam. The $B B R$ depends also upon the bubble diameter squared. We had to aim at diameters small enough to keep the confusion region near the production vertex as small as possible for efficient detection of short-lived particle decays. The reference intensity $\left\langle I_{\mathrm{r}}\right\rangle$ is the other non-geometrical factor, but this also leaves little flexibility for adjustment.

We used a commercially available, powerful holographic ruby laser (JK 2000, Lumonics Inc.), for which we developed a pulse stretching circuit [22]. The laser was installed in an air-conditioned clean room. An optical relay system was used to transport the beam $29 \mathrm{~m}$, mostly in vacuum, to the bottom of the bubble chamber [18]. Its quality (light pulse energy, its width and shape, and the spatial intensity distribution) was monitored before entrance into the chamber liquid [23].

The beam entrance window was made as an aspheric light-dispersing lens, which acts as a sophisticated beam splitter. Only the small axial part of the light serves as the reference beam and exposes completely the $70-\mathrm{mm}$ film format, hitting the film perpendicularly within $1^{\circ}$. The rest of the beam illuminates the tracks within a conical volume having a half-angle of $30^{\circ}$. The intensity of this illumination beam is designed to increase at large angles to partially compensate for the decrease of the light scattered by the bubbles at these angles [18]. The holographic volume overlaps the conventional fiducial volume.

During data-taking the bubble chamber has to be synchronized with the particle pulses from the accelerator. The timing is adjusted so that beam particles arrive near the pressure-minimum of the expansion curve, and provides appropriate delays before illuminating the tracks for holography, the high-resolution and the four conventional cameras. The growth of bubble diameter follows a square root of time law. The three delays were chosen as $1 \mathrm{~ms}, 1.5 \mathrm{~ms}$ and $10 \mathrm{~ms}$. The chamber operating conditions were adjusted so that good visibility of tracks for these delays was obtained. The duration of the beam spill from the accelerator is of the order of a couple of milliseconds, during which billions of neutrinos pass through the chamber liquid. An interaction of one (or two) of these neutrinos can occur any time during this interval. Precise timing of these interactions is needed in order to adjust the critical delay for taking the hologram, whereas the relatively small change for the standard optics is less significant for the quality of the track photos. The interaction time is measured online from two types of electronic detectors downstream of the chamber.

The replayed hologram of particle tracks gives three-dimensional information. However, to find an event vertex using the hologram alone would require an extremely timeconsuming focusing search over the entire depth of field, which is almost $4 \mathrm{~m}$. Therefore, the spatial co-ordinates of vertices expected to lie in the holographic volume are first measured on the conventional pictures, and then the computer-controlled replay machines (Hawaii replay machine [24], HOLRED [25], Fermilab replay machine [26], HAM [27]) are provided with this geometry information, substantially accelerating the search for the position in space. The replay gives either a real image using a time-reversed illumination $[24,25,27]$ or a virtual image with a time-forward illumination [26].

\section{DATA-TAKING}

The E-632 experiment was subdivided into two run periods, interleaved by tests of the holographic set-up in the empty, warm chamber. The first part of the run in 1985 could be considered as a learning process during which many technical problems 
were uncovered and analysed in detail [28], [29]. The resulting modifications led to the successful final run. The warm tests revealed that, during the first run, multiple-reflected illumination beam laser light was reaching the film and overwhelming the reference beam [see Eq. (4)]. Several hundred light-absorbing baffles were installed according to detailed light-pass calculations, thus preventing stray light from reaching the holographic film via multiple reflections. During the second part of the physics run in 1987/88, 293060 conventional 3-view pictures and $\sim 218000$ holograms, of which $\sim 110000$ are useful for physics analysis, were recorded simultaneously. We discuss in detail only this part of the experiment and make ample reference to previous publications.

In spite of many improvements to the system, holograms still differed in quality owing to various changes in the operating conditions of the chamber, the laser performance, or film development. For the present analysis we use 656 holograms that contain a visible neutrino-induced event.

\subsection{The chamber operation}

The bubble chamber was filled with a $62 / 38 \mathrm{~mol} \% \mathrm{Ne} / \mathrm{H}_{2}$ mixture. This ratio was kept stable during the run to better than $\pm 1 \%$. The operating temperature of the chamber was $29.6 \pm 0.1 \mathrm{~K}$ and the temperature gradient from bottom to top was kept smaller than $0.1 \mathrm{~K}$. The usual expansion ratio of $0.4 \%$, i.e. change of volume to total liquid volume, could be varied by changing the piston stroke to make minor adjustments to the desired bubble densities and growth rates of bubbles. The latter variations control the degree of superheat and probably have the largest effect on the quality of holograms as far as the chamber operation itself is concerned. The duration of the expansion cycle was approximately $80 \mathrm{~ms}$.

\subsection{The neutrino beam}

The Fermilab Tevatron delivered three spills separated by $10 \mathrm{~s}$ followed by a 40s pause per each accelerator cycle of $60 \mathrm{~s}$ to the bubble chamber. Each neutrino pulse had an approximately Gaussian intensity distribution versus time with a base width of $\sim 3 \mathrm{~ms}$. This pulse was injected during the expansion of the chamber liquid at or slightly before the pressure minimum. The neutrino beam was spread geometrically over the entire chamber, falling off to the sides and having a $\pm 0.5 \mathrm{~m}$ fwhm.

\subsection{The laser performance}

The high-power pulsed ruby laser with a wavelength of $694 \mathrm{~nm}$ (JK 2000, Lumonics Inc.) is described in Refs. [17, 30], and the latest version of the pulse stretching circuit in Ref. [22]. The monitoring of the spatial shape and position of the laser beam at the chamber entrance port is discussed in Ref. [23]. The pulse-to-pulse variation of output averages $10 \%$, which is typical for pulsed lasers. The pulse duration was chosen to be between 4 and $7.5 \mu \mathrm{s}$, with an average pulse width variation $\leq 1 \%$. More difficult to control was the flatness of the pulse, which showed modulations of $30 \%$. These quantities were recorded on a pulse-by-pulse base by the bubble chamber computer together with the timing of the events (see 3.8).

\subsection{Optical filters}

A red colour filter (RG 665) was installed in front of the holographic film as well as filters with a complementary colour (BG 39) in front of the conventional camera flashtubes in the other ports. These filters prevented the flash-tube light from exposing the 
holographic film. Exposure of the conventional film by laser light was not a problem at the present energy level, due to the high $f$ number of the conventional lenses $(f / 12)$, the presence of a Wratten 58 colour filter in each lens, and the low sensitivity of the Kodak 2482 film to the deep red ruby laser light. Four fast photodiodes were mounted on the platen next to the holographic film to monitor the reference beam intensity.

\subsection{The laser beam profile}

The oscillator stage of the JK 2000 laser produced a TEM T0 $_{0}$ output (Gaussian beam profile), which then passed through three amplifiers, the lenses of an optical relay system, and was reflected by several mirrors, to arrive at the dispersing lens. There, the spatial beam profile should ideally be flat, since this lens was designed for such an input. A reconstructed profile, covering the 5-cm diameter of the dispersing lens entrance, is shown in Fig. 4, see also Ref. [23]. Apart from the somewhat spotty character, the typical variation of intensity across the beam profile corresponded to around $\sim 20 \%$ of the mean value.

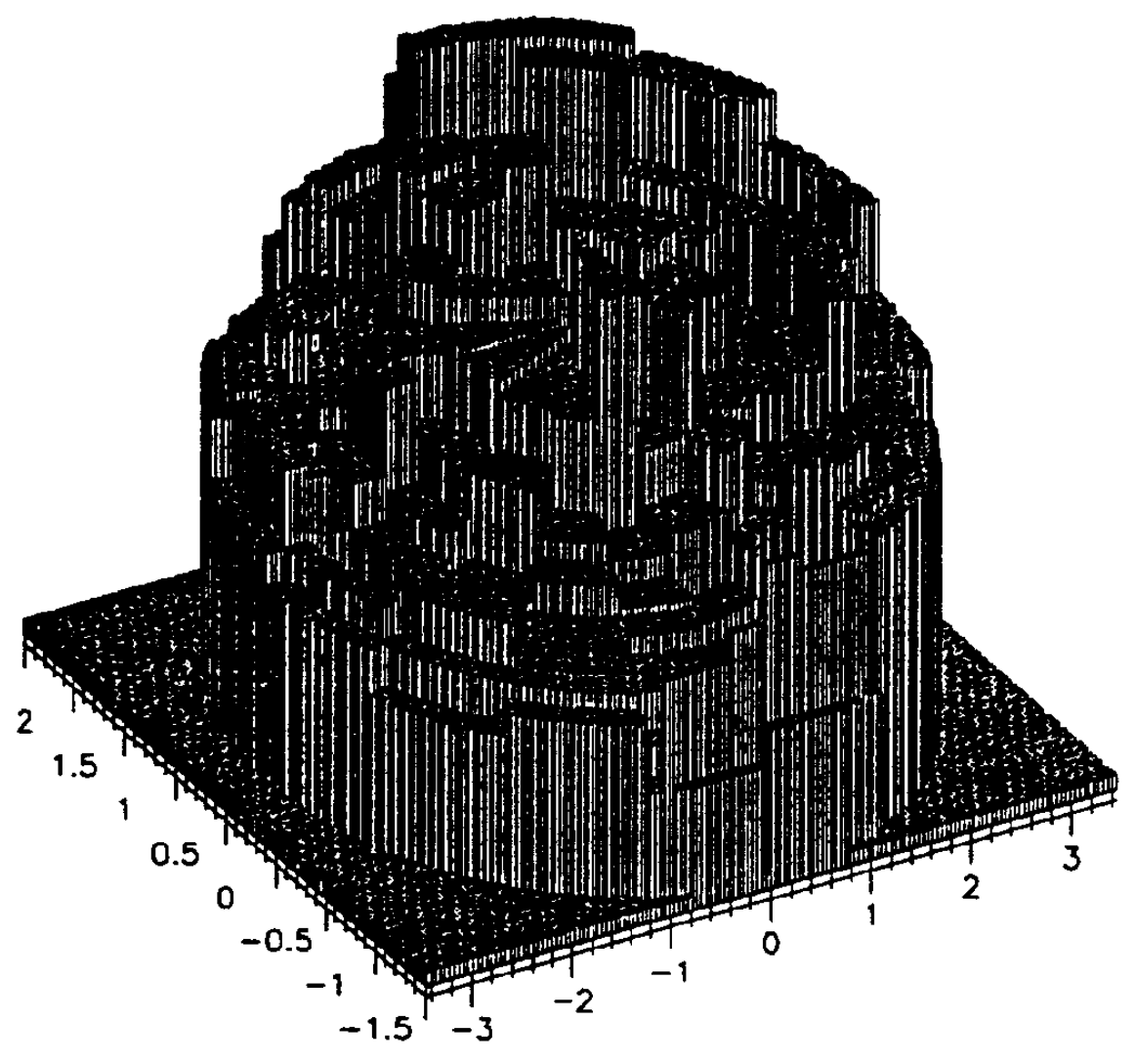

Figure 4: Example of an off-line reconstruction of the laser beam profile. Dimensions are given in $\mathrm{cm}$.

\subsection{Design of the dispersing lens}

A detailed description of the two-element dispersing lens is given in Ref. [18] (Fig. 5). The design was governed by the requirements to avoid excessive heating of the liquid and to illuminate the maximum possible with a good quality laser beam. The design values of 
the illumination intensity $F\left(\Theta_{\mathrm{i}}\right)$ per solid angle as function of the illumination angle $\Theta_{\mathrm{i}}$ is shown in Fig. 6. The lens shape was optimized for a $B B R=0.33 \times 10^{-7}$ (see Section 5).

The fringe visibility and the exposure level determine whether objects can be detected at the reconstruction stage. If it is assumed that bubbles with a fringe visibility $V$ (Eq. 3) larger than a certain minimum fringe visibility $V_{\min }$ can be seen, the visible holographic volume can be calculated. The visible holographic volume is expected to depend only upon the $B B R$. The result of a theoretical calculation showing the expected visible volume versus the minimum detected beam branching ratio $B B R_{\min }$ is given in Fig. 7 . It shows that the visible volume had been optimized for a $B B R_{\min }=0.33 \times 10^{-7}$. The corresponding holographic volume is $4.7 \mathrm{~m}^{3}$. The actual distribution of the light during the exposure of the chamber is not well known.

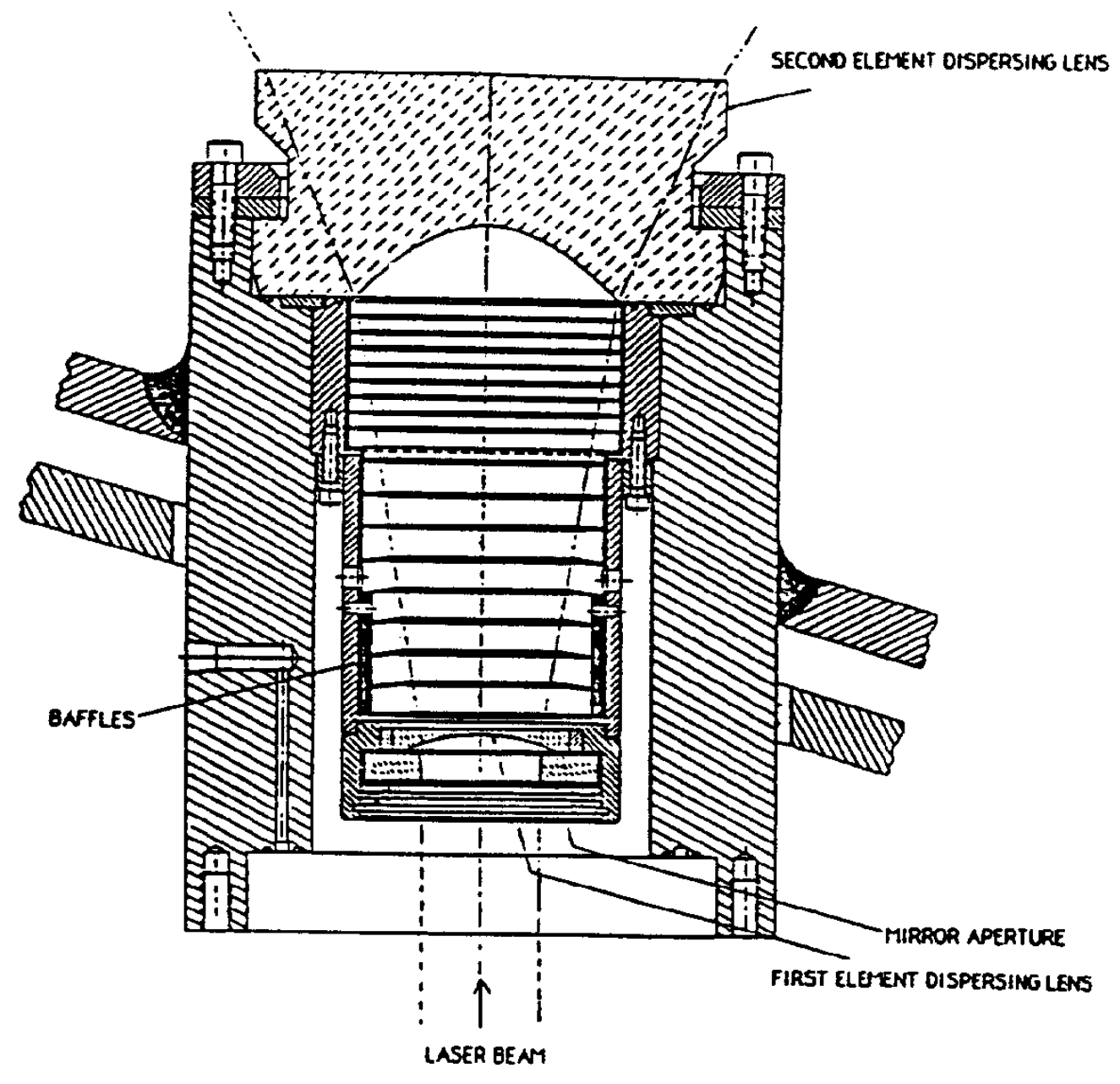

Figure 5: Drawing of the two-element dispersing lens. 


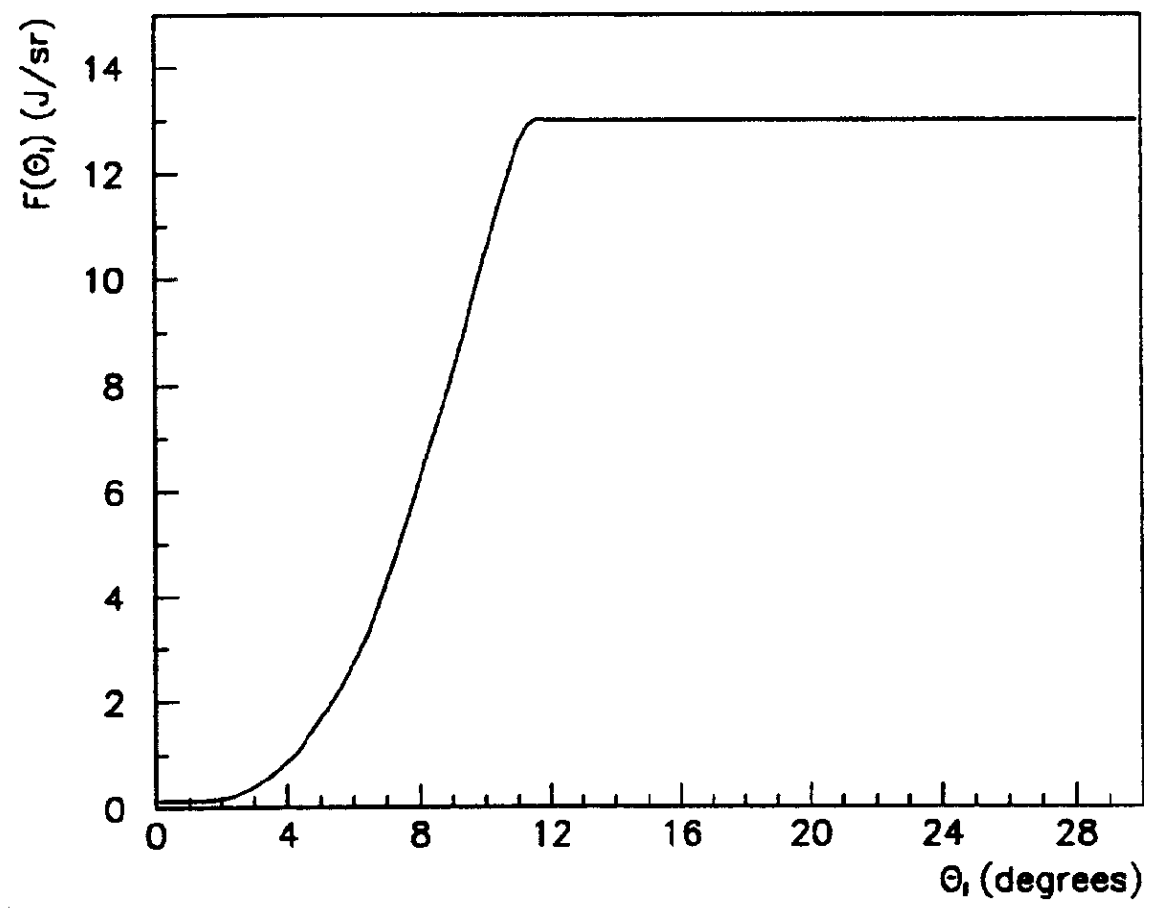

Figure 6: The illumination intensity distribution $F\left(\Theta_{\mathrm{i}}\right)$ per solid angle as a function of the illumination angle $\Theta_{\mathrm{i}}$.

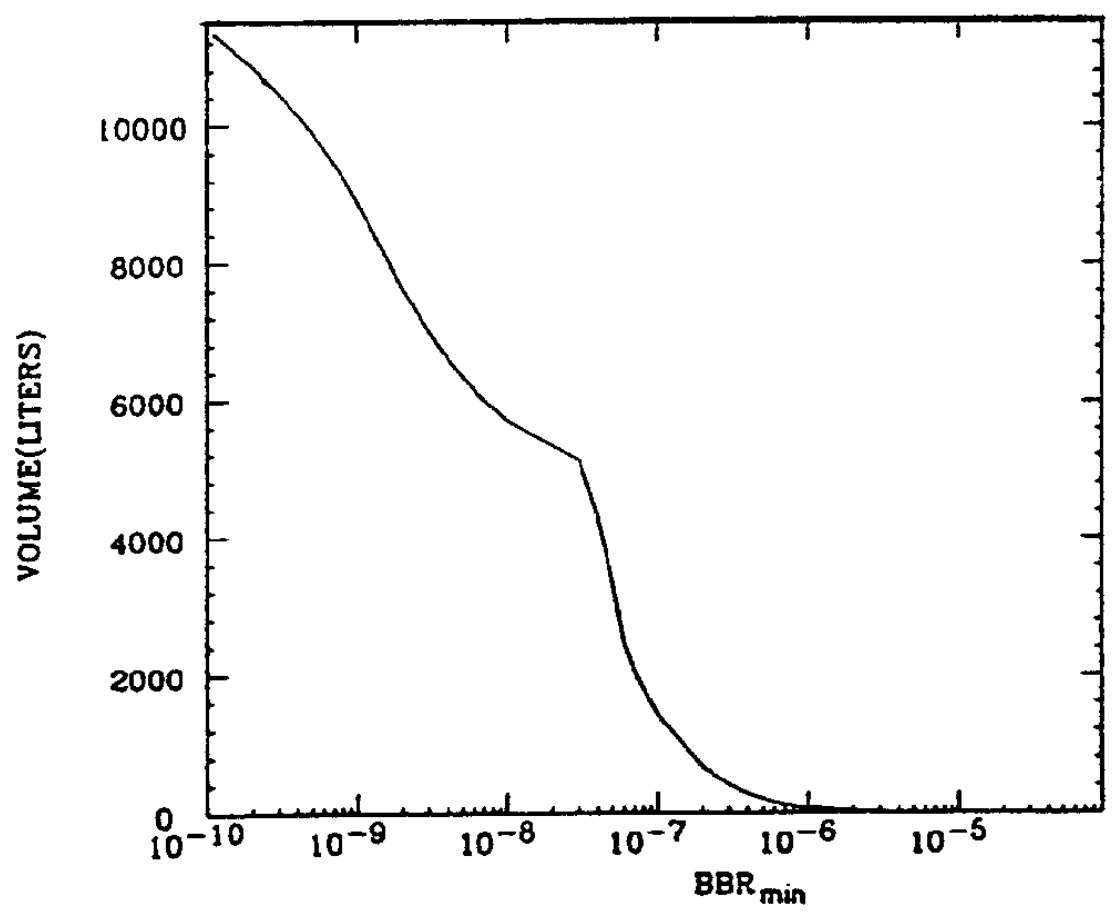

Figure 7: The visible holographic volume versus the minimum-detectable beam branching ratio $B B R_{\min }$. 


\subsection{Heating effects of the liquid}

The two-element dispersing lens was designed so as to spread the laser beam geometrically at its entrance into the liquid to reduce its energy density per unit area. The aim of the pulse stretching was to distribute the instantaneous power over a long time interval. By these means we suppressed (almost) completely macroscopic boiling within the bulk of the liquid, which would have affected adversely the quality of the conventional pictures. However, we were less effective in the prevention of microscopic boiling close to the laser beam entrance into the liquid. This microscopic boiling is initiated by the laser pulse itself and affects the holograms. Its presence became evident from comparison of the time structure of the laser pulse before the entrance into the chamber with its structure at the film plane (Fig. 8). Any difference between the two slopes of light intensity versus time was an indication for the presence of either a heated layer of liquid or microscopic bubbles near the lens surface. Owing to scattering from growing micro-bubbles, more light from the object beam reaches the holographic film and increases its exposure in a noncontrollable way. This boiling destroys the point-source feature of the reference beam, and thus deteriorates the quality of the hologram.

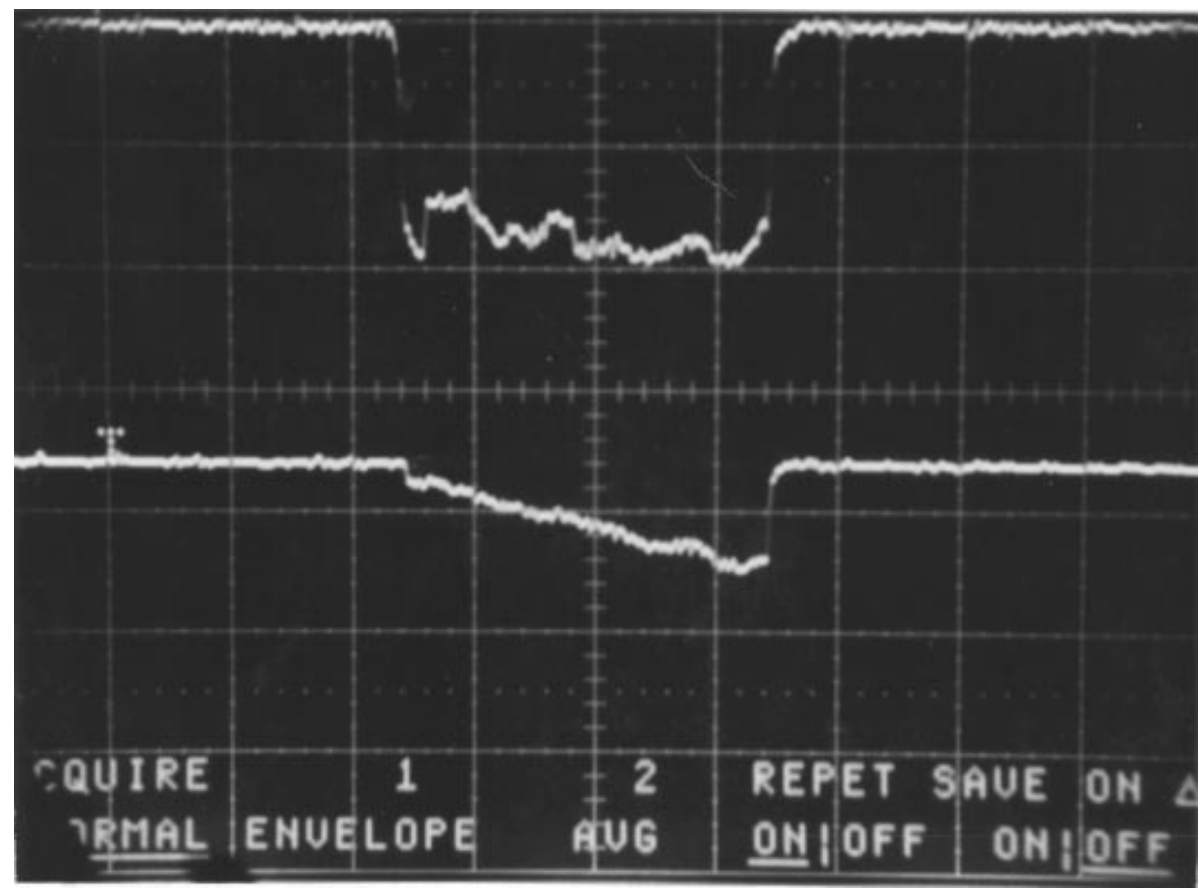

Figure 8: Oscilloscope picture of light intensities (arbitrary units) of a laser pulse versus time. Upper trace: measured downstream of the laser amplifier. Lower trace: measured by means of the photodiodes on the holographic film platen. Horizontal scale: $0.5 \mu \mathrm{s} /$ div.

\subsection{Timing of the holograms}

The radii of bubbles in the liquid grow according to a square root of time law. For a chosen liquid temperature and expansion ratio this growth rate and the bubble density are fixed. We aimed at an operating condition such that bubbles attain a diameter of $\leq 120 \mu \mathrm{m}$ in space at $1 \mathrm{~ms}$ after their creation, and also are compatible with sufficient track quality (visibility) for the conventional cameras. To be able to obtain this size consistently during the hologramming we need to know the interaction time of the neutrino. A trigger logic was developed using the External Muon Identifier (EMI, [31]) and the Internal 
Picket Fence (IPF, [32]) to signal the occurrence of a neutrino event with a precision of $1 \mu \mathrm{s}$. This trigger was then used to initiate the laser firing sequence. If no event trigger occurred during the first $1.3 \mathrm{~ms}$ (1.7 ms during the second part of the run in 1987/88) of the spill, a default trigger was generated. Holograms were taken $1 \mathrm{~ms}$ after this event trigger.

\subsection{Bubble movement, growth, and vibrations}

Vibrations and any movements of bubbles due to turbulence in the liquid and/or buoyancy effects, as well as the change in size during the laser pulse diminish the quality of the hologram, as has been discussed in Ref. [18]. A compromise had to be found to minimize the boiling effect by lengthening the pulse and to keep the displacement of bubbles sufficiently small by using an appropriate short pulse. We selected laser pulse duration of $4-7.5 \mu$ s at an energy level of $6 \mathrm{~J}$.

\subsection{The magnetic field}

Linearly polarized light is subject to the Faraday effect when it passes through a magnetic field. Assuming the Verdet constant to be similar in liquid hydrogen and liquid neon/hydrogen $\left(\sim 5 \times 10^{3} \mathrm{~min} / \mathrm{T} \cdot \mathrm{cm}\right)$, we expect that, in the field of the 15-Foot Chamber of $3 \mathrm{~T}$ and a path length of the light of some $400 \mathrm{~cm}$ in the liquid and $9.0 \mathrm{~cm}$ in glass and quartz, the polarization would be rotated by about three complete revolutions. However, the rotations depend on $\mathbf{B} \times \ell$ ( $\ell$ is the path) and both the reference beam and the scattered beam have, to a good approximation, the same $\mathbf{B} \times \ell$.

\subsection{Holographic film development}

An upper limit for the laser energy available to expose the AGFA-GEVAERT holotest film 10E75 was given by the admissible heating of the bubble chamber liquid and by the performance of the pulse-stretched laser. The sensitivity of the film had to be augmented by adding phenidon to the KODAK D19b developer, which did not visibly degrade its resolution. We were obliged to use a fairly low density of 0.6 , just 0.4 over the fog density. This low exposure meant a loss of resolution, since we could observe with reasonable contrast only bubbles with $\sim 120 \mu \mathrm{m}$ diameter instead of the theoretically possible $70 \mu \mathrm{m}$. We did not bleach the holograms, which could have resulted in a somewhat improved resolution.

\subsection{Holographic replay}

After their recording the holograms have to be replayed for physics analysis. Virtualand real-image replay machines were built in our collaboration for the present experiment. Their designs and performances are described in detail in Refs. [24]-[27]. Essential for the quality of the replay machines are the alignment of the optical elements and sufficient light intensity. The wavelength of the laser for the replay should be ideally the same as the ruby light $(\lambda=694 \mathrm{~nm})$. Dye lasers were used for HOLRED and the Hawaii replay machine, a $\mathrm{Kr}^{+}$ion laser for Rutgers $\operatorname{HAM}(\lambda=647 \mathrm{~nm})$, and a continuous neon/helium laser $(\lambda=633 \mathrm{~nm})$ for Fermilab during replay. A Vidicon camera or photographic film with high resolution were placed as detectors in the image plane.

\section{ANALYSIS OF PHOTOGRAPHS}

The primary aim of the holographic part of the experiment is to investigate the production and decay of short-lived particles, i.e. to search for short decay lengths near 
the neutrino interaction. First, the conventional film is scanned and the positions of all neutrino event vertices are measured and recorded. Next, the position in holography replay space is calculated for those events in the cone illuminated by the laser beam. Finally, only the holograms for these events are examined in detail, by making a systematic search starting at the calculated position of the vertex. Some of these events are not found in the hologram, for which there may be a variety of reasons, such as the failure of equipment or maladjustment of one or more of the parameters discussed in the previous section.

Entire rolls (a roll has $\sim 1000$ frames) are discarded from further analysis when:

1) during the pre-scanning films were judged to have insufficient holographic quality, i.e. even cosmic ray tracks or reflections from the dispersing lens were poorly visible, mostly due to micro-boiling;

2) the holographic film was incorrectly developed;

3 ) the holographic film was over- or underexposed (average density $<0.5$ or $>1.2$ ); and individual frames with events were obviously not counted when

4) the laser did not fire.

This procedure reduced the total number of frames to be investigated in detail from 218000 to 110 000. The latter were then further subdivided into good and fair rolls according to more subjective criteria.

The co-ordinate system for conventional photographs has its origin in the centre of the bubble chamber, see Fig. 1. The holographic co-ordinate system is inclined and has its $z$-axis pointing from the holographic camera to the dispersing lens, with $z_{\text {holo }}=0$ at the film plane. An algorithm was developed for the co-ordinate transformation.

The analysis of the 110000 good and fair holograms continued along the following lines: All events from the conventional film, having their vertex co-ordinate in the illumination cone of the laser with the half-angle of $30^{\circ}$ and scattering angles $\alpha<40^{\circ}$ and found in the hologram, were plotted in the holographic co-ordinate system as 'seen' events. The positions of the 'unseen' events were also registered. Figure 9 shows as an example the distribution of seen and unseen events (one holographic roll) in a scatter plot of the holographic $z$-axis versus illumination angle.

Reasons for not seeing an event in the hologram are manifold. The most obvious one is that the event is too close to the reference beam, so that a replay suffers from excessive light intensity in this direction. We therefore exclude from further analysis a cone with an half-angle of $1^{\circ}$ from the laser axis. This corresponds to a loss of $0.05 \mathrm{~m}^{3}$ in volume. Various other causes may contribute to the non-visibility of events:

a) the laser pulse came too early, when the bubbles did not yet have the minimum size to make them visible holographically;

b) the Beam Branching Ratio $(B B R)$ was too low;

c) there was too much boiling on the dispersing lens;

d) the bubble chamber conditions (temperature, expansion ratio) resulted in a too small bubble growth rate and/or density.

We have to arrive at a clean sample of events to get the desired information on the $B B R$. Therefore, we have to discard all events that do not have the correct timing (a). That is done with the information from the electronic counters surrounding the bubble chamber. It explains some non-visible events. It also helps to eliminate the seen events having very fat tracks, i.e. when the laser pulse came too late.

The next step in the analysis has to take into account the combination of effects (b) and (c). This helps to explain the non-visibility of other events. A check of the bubble chamber operating conditions follows. The difficult and most interesting 
part of the analysis is then the study of the scattering angle, combined with the illumination intensity at the place of the event vertex, which will lead us to find 'the minimum-detectable $B B R$ '. The factor $k$ in Eq. (4) was considerably reduced between the two run periods, but its absolute value for the second period under operating conditions could not be determined.

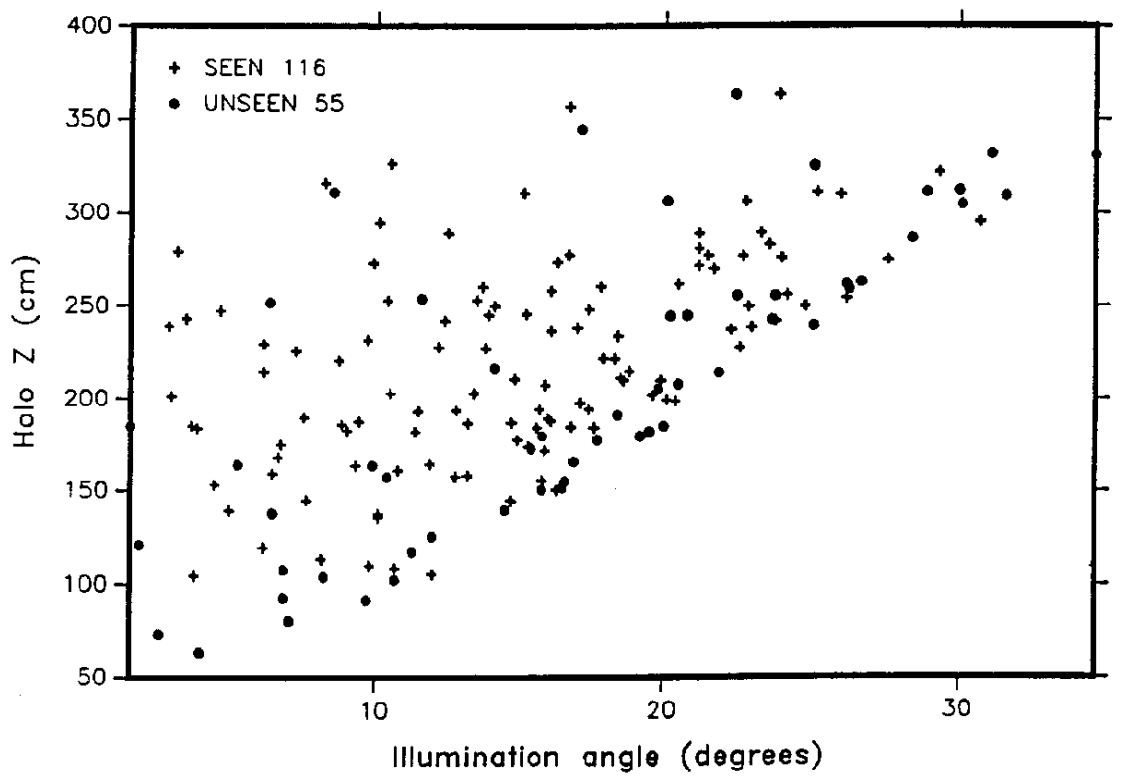

Figure 9: Scatter plot of seen and unseen events (one holographic roll): holographic zaxis versus illumination angle; $z$ is the distance from the film, measured along the line connecting film and lens.

\subsection{The bubble size}

Knowledge of actual bubble size when the hologram is taken is critical in determining the $B B R$ [see Eq. (2)]. There are several approaches to determine the actual bubble size when the hologram is taken.

One approach is based entirely on the theory of bubble formation and growth, where the diameters can be calculated as a function of growth time, provided all thermodynamic data for the operating conditions of the bubble chamber are well known. The theory has been tested in various pure liquids [33]-[37]. Unfortunately, the relevant thermodynamic data for our specific $\mathrm{Ne} / \mathrm{H}_{2}$ mixture are not known with sufficient accuracy. Therefore we have to rely on various kinds of measurements.

Selected experimental results are available for some mixture ratios from a smaller bubble chamber [34], but not for the specific mixture used in our experiment. Of particular interest are measurements in two mixture ratios, one with slightly lower, the other with somewhat higher neon content. They provide guidance for what we should expect for our mixture ratio.

We are constrained to use the square-root-of-time dependence for bubble growth together with the measured image sizes on the pictures taken with the conventional cameras and the high-resolution camera, and to extrapolate to the time when the hologram is taken. Beam injection time gives us the origin (creation of the bubble), and the short and long delays for the high-resolution and the conventional pictures, respectively, give additional points to define the curve. The short delay for the hologram is known and 
the corresponding bubble size should be on the curve(s). The diffraction limit of the two conventional optics will, however, make this analysis more difficult, as will be discussed later on.

The bubbles are transparent objects, having a steep temperature gradient in the liquid close to the vapour surface that produces a change of the refractive index. Therefore, bubbles can act as lenses, so it is difficult to know their actual size [36]. The general consensus is to define the size of a bubble as the full width at half-maximum of the image on the film and to scale this quantity with the demagnification factor of the optical arrangement. A less used technique is to estimate bubble diameters by contrast measurements.

We aimed during our neutrino experiment at the highest possible bubble density in order to get optimum information about possible small kinks along the tracks. This density decreases with time due to the coalescence of growing bubbles. Individual bubbles are optically almost non-separable from each other at any of our time delays.

Other difficulties in the determination of the size originate from the recording material, its grain size, exposure intensity, and development of the exposed film. Whereas fwhm values of the apparent bubble size are largely independent of exposure intensity in bright-field illumination [38, 39], recording in dark-field illumination depends heavily on the intensity and the subsequent photographic development. The latter problem needs special attention when measurements of bubbles sizes are made on holograms, which have to be replayed and recorded on film. The speckle in holograms diminishes the contour of the bubble image. We return to this problem in more detail below.

Geometric factors can cause asymmetric bubble images. Non-normal illumination of the film can cause half-moon-shaped images ([36], Fig. 10) or variable size aspheric bubble images.
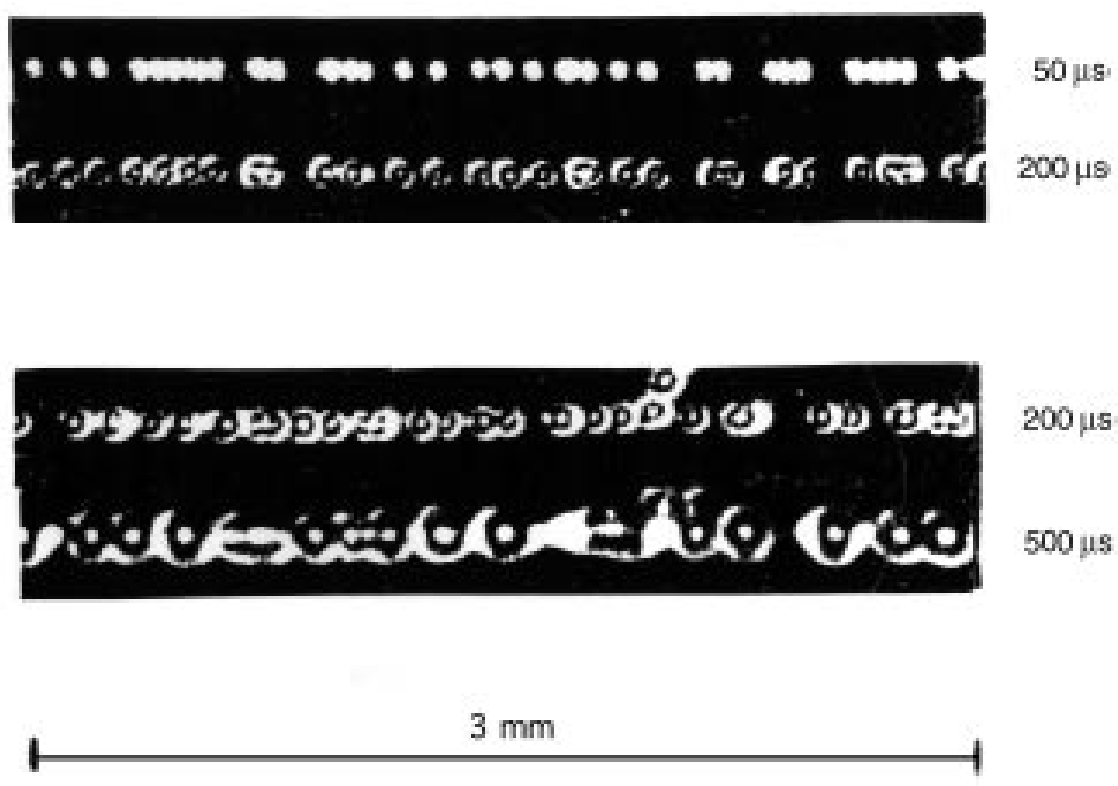

Figure 10: Photos of tracks taken with different flash delays in a propane bubble chamber with a 1:1 magnification optics: (a) a track at a flash delay of $50 \mu \mathrm{s}$ and of $200 \mu \mathrm{s}$; (b) a track at a flash delay of $200 \mu$ s and of $500 \mu \mathrm{s}$. 
In spite of the many sources of uncertainty, measurements on the same track (ideally the same bubble) from the standard and high-resolution optics can provide an estimate of the bubble size when the hologram is taken. The description of three experimental procedures follows.

\subsubsection{Bubble size from track width}

Measurements of bubble size were made with a microdensitometer directly on the bubble chamber film. To compare these bubble sizes each result must be corrected by using the appropriate magnification factor. The demagnification at the intersection of the optical axis with the mid-plane of the bubble chamber film to chamber space is $\sim 1 / 55$ for standard cameras and 1/15 for the high-resolution camera. The optical axes are inclined and intersect the mid-plane at different points. We used an Automatic Recording Microdensitometer (Joyce Loebl, MK3CS). Its main properties are exchangeable, calibrated density wedges (providing information about density differences), filters of known densities for absolute calibration, adjustable slit width and height, and speed of the table on which the film is attached. We adapted these parameters to our track and background (fog) densities and the expected 'bubble size'.

The slit was either (a) perpendicular to the track and moved along it, or (b) parallel to the track and moved across it. Both movements have inherent error sources and tend to overestimate the size of an individual bubble. In case (a), owing to the high density of bubbles along the track, most of them had (partially) grown together at the instant of recording, so that the background level in the gaps between bubbles is difficult to determine. In case (b) small $\delta$-electrons or small jitters of bubbles along the straight trajectory of the particle widen the track. Furthermore, the (slight) curvature, present in even small track segments does not allow for a perfect parallel alignment of track and slit.

For our analysis we selected a neutrino interaction (roll 2346, frame 737), which was clearly visible in all conventional views, in the high-resolution view, and on the hologram. It also had the correct timing for the hologram (EMI information). The vertex occurred close to the mid-plane and centre $(x=15.78 \mathrm{~cm}, y=-12.27 \mathrm{~cm}, z=8.93 \mathrm{~cm})$ of the bubble chamber, so that the standard optics and the high-resolution camera recorded it in best focus. Tracks remain mainly in a horizontal plane. The plane of best focus is curved, so tracks down- or upstream widen due to defocusing. Figures 11(a), 11(b), and 11(c) show the corresponding photographs. We studied (i) a track emerging almost perpendicular to the beam direction close to the vertex of the event, (ii) the two branches of a close-in strange particle (Vee-vertex : $x=18.16 \mathrm{~cm}, y=-11.35 \mathrm{~cm}, z=9.21 \mathrm{~cm}$ ), as indicated in Fig. 11(d), and (iii) the muon track from the event vertex far downstream. The choice of these tracks and distance from the neutrino vertex was dictated by the technical feasibility of measuring individual tracks with the microdensitometer slit in both the parallel and perpendicular directions to the tracks without interference from neighbouring tracks.

The selection of track sections for the measurement is also constrained by the holographic replay. Bubble tracks from neutrino interactions can be replayed in best focus only over a small section before running out of focus. Since we are mainly interested in the decay of short-lived particles, which occur close to the interaction vertex, we selected track segments close to the vertex or near the origin of the strange-particle decay. This gave us the advantage of identifying the same track segments (almost the same sequence of bubbles) on the conventional and high-resolution film.

Bubble densities depend upon the kinetic energy divided by the rest mass of the particle. These densities are high for slow particles, passing with increasing energy through 
a minimum and showing afterwards a relativistic rise (see e.g. Ref. [37]). For slow particles, therefore, more bubbles have the chance to grow together and the track size appears to be larger. We made some calibration measurements on a stopping proton, which was the easiest track to measure since it was almost perpendicular to the neutrino beam and to the $\sim 20$ tracks going in the forward direction.
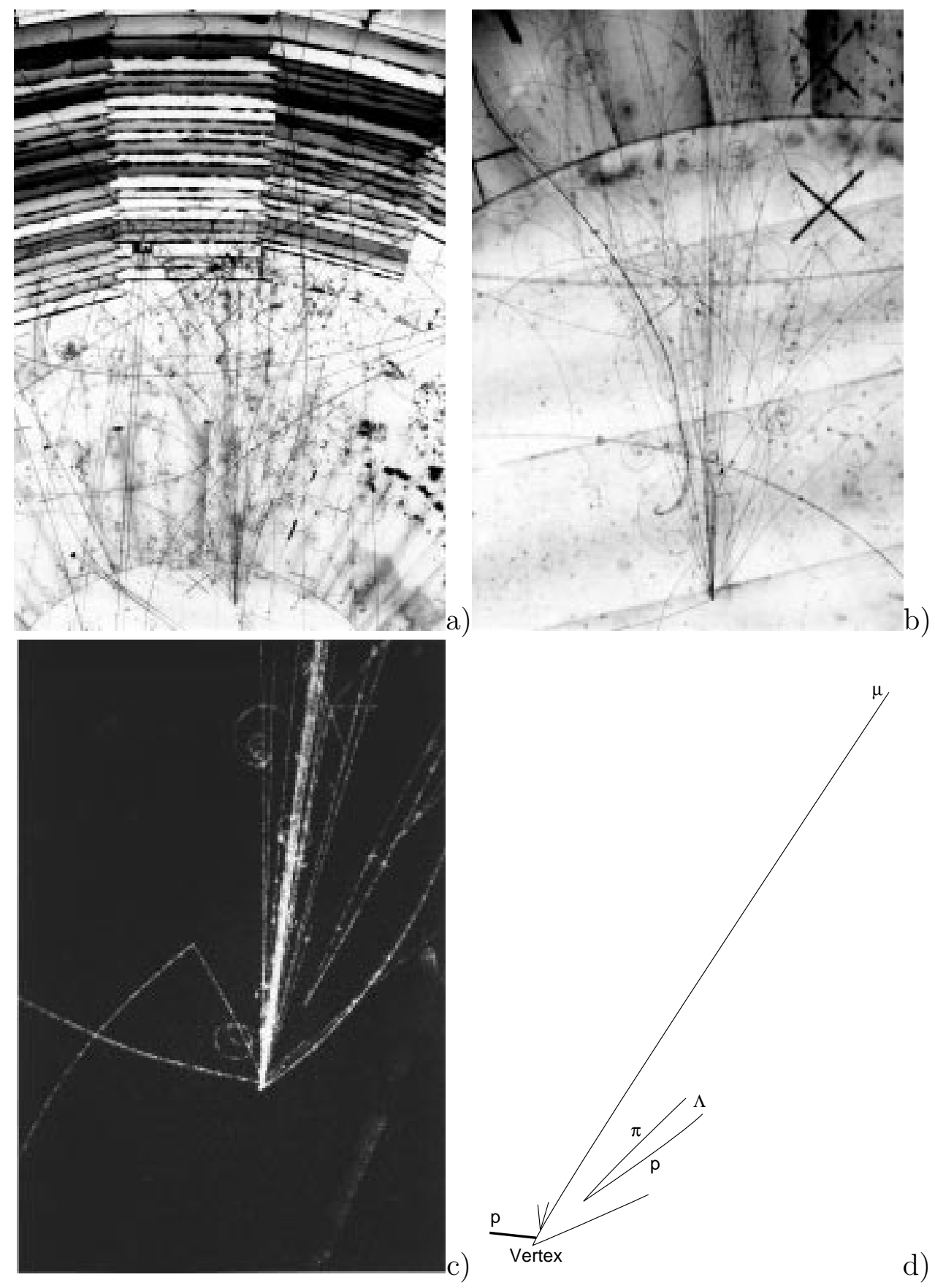

Figure 11: Photographs of a high-energy neutrino event: (a) photo taken with one of the standard optics (view 6); (b) photo taken with high-resolution optics; (c) hologram replay using exposure 1 Fig. 12; (d) schematic drawing of the parts of tracks measured with a densitometer. 
The replayed hologram was investigated from photographs taken with the Fermilab replay facility with four different exposure times [Figs. 12 (exposures 1-4)]. The demagnification factor of the photos of replayed holograms (Figs. 12) to real space was determined from the event vertex distance to Vee-vertex to be 4.9. The paper print [Fig. 11(c)] of photo 12 (exposure 1) gives visually the best resolution and its quality would be preferred during physics analysis of the interaction. We measured the same track segment at all four holographic replay exposures and found the best reproducible results with the smallest error bars on the track widths from photo 12 (exposure 2). With the exception of the lowest exposure [Fig. 12 (exposure 1)], increasing optical density did not significantly increase the measured bubble size (Table 1). Applying the magnification factor film-to-chamber, this corresponds to $105 \pm 24,213 \pm 45,181 \pm 16,224 \pm 117 \mu \mathrm{m}$ bubble diameters.

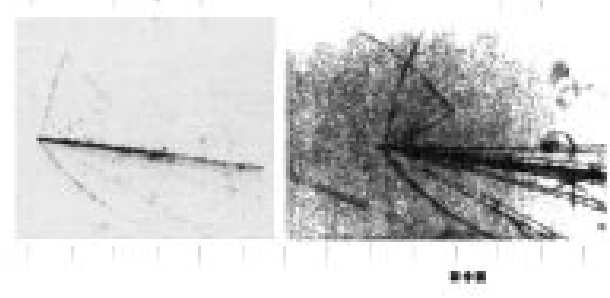

2

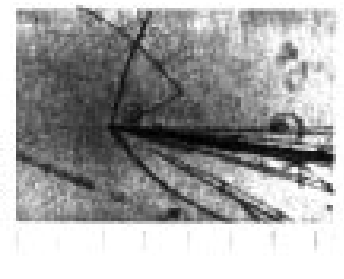

3

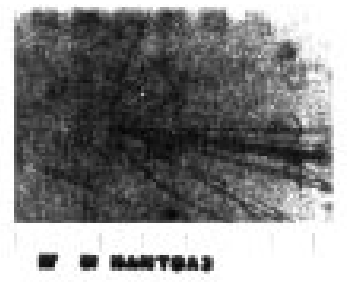

4

Figure 12: Photographs of the replayed hologram using different exposure times.

Table 1: Measurements on film on three sections of the stopping proton on four different replay exposures of the hologram. Values in brackets are sizes in chamber space

\begin{tabular}{|c|c|c|c|c|c|}
\hline $\begin{array}{l}\text { Distance } \\
\text { from vertex } \\
(\mathrm{mm})\end{array}$ & $\begin{array}{l}\text { Exposure } 1 \\
\text { track width } \\
(\mu \mathrm{m})\end{array}$ & $\begin{array}{l}\text { Exposure } 2 \\
\text { track width } \\
(\mu \mathrm{m})\end{array}$ & $\begin{array}{l}\text { Exposure } 3 \\
\text { track width } \\
(\mu \mathrm{m})\end{array}$ & $\begin{array}{l}\text { Exposure } 4 \\
\text { track width } \\
(\mu \mathrm{m})\end{array}$ & $\begin{array}{l}\text { Average } \\
\text { track width } \\
(\mu \mathrm{m})\end{array}$ \\
\hline 0.21 & 53 & 113 & 130 & 67 & $91 \pm 30$ \\
\hline 0.94 & 87 & 117 & 97 & 240 & $137 \pm 60$ \\
\hline \multirow[t]{3}{*}{1.56} & 57 & 173 & 107 & 117 & $113 \pm 41$ \\
\hline & $66 \pm 15$ & $134 \pm 28$ & $114 \pm 10$ & $141 \pm 73$ & \\
\hline & $(105 \pm 24)$ & $(213 \pm 45)$ & $(181 \pm 16)$ & $(224 \pm 117)$ & \\
\hline
\end{tabular}

The width of the image of this stopping proton was measured in the high-resolution view (HRC), and two conventional views (CC) close to the vertex, and on a track segment near its stop. The increase of the ionization density is seen, as well as the effect of its large dip angle $\left(30^{\circ}\right)$. The determination of fwhm depends upon the determination of background level and is particularly sensitive in holograms (speckle). Results of these measurements, including those of lower background assumption for the holograms (not given in Table 1) are shown in Fig. 13. This is an upper limit for bubble size, as confirmed by the other two analysis techniques to be discussed below.

The main emphasis of the analysis was on the measurement of track size (and where possible bubble density) of the pion and proton from a strange-particle decay and the muon of the charged-current event. The measurements of the inner branch of the Vee from the standard camera, the high-resolution, and the hologram are shown in Figs. 14(a), 
14(b), and 14(c), respectively. The muon from this charged-current interaction could be measured far downstream [Figs. 15(a) and 15(b)]. An 'early' muon happened to be on the same photograph. Its arrival time is $1 \mathrm{~ms}$ earlier than the neutrino interaction, and it had therefore more time for the bubbles to grow. It was also visible on the film from the high-resolution camera [Figs. 16(a) and 16(b)].

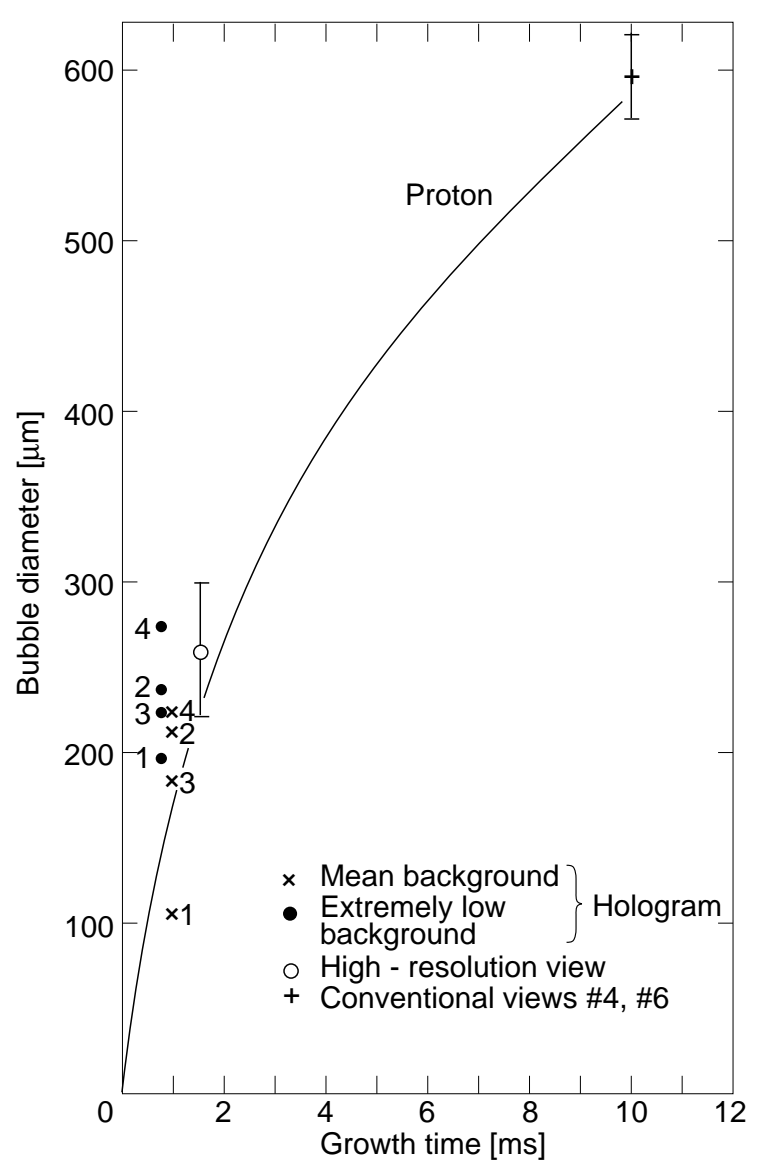

Figure 13: Results from track-width measurements on the proton: hologram (1 ms delay): $\times$ mean background (see Table 1), · extremely low background; high-resolution camera (delay $1.5 \mathrm{~ms}$ ); conventional cameras \#4 and \#6 (delay $10 \mathrm{~ms}$ ). The solid line is the $\sqrt{t}$-dependence using the measured diameter at $10 \mathrm{~ms}$ to guide the eye.

Measurements near the entrance and exit points of the early muon show an increase in width, which can be explained by the track leaving the region of best focus.

Results of measurement of track widths (fwhm) from CC and HRC, taken in brightfield illumination, are summarized in Tables 2 and 3 (first 3 columns). Errors on the bottom of the columns are statistical only. Systematic errors will be estimated later together for all three measuring methods.

In Fig. 17 bubble diameters as function of growth time are presented, including those from the hologram. As a guidance to the eye the square root of time dependence is shown, based on the value of the mean track diameters at $10 \mathrm{~ms}$ (muon, proton and the two branches of the Vee). 


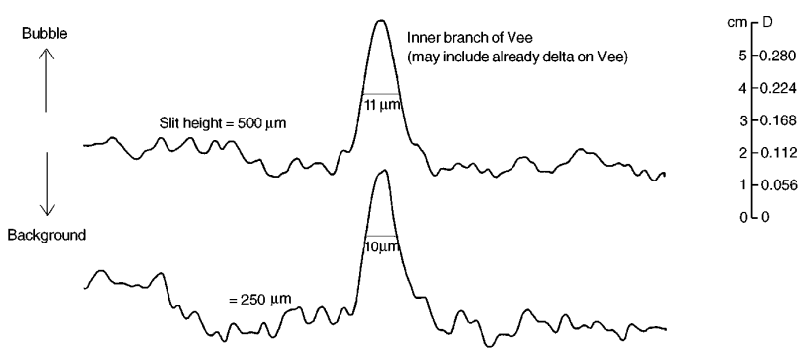

a)

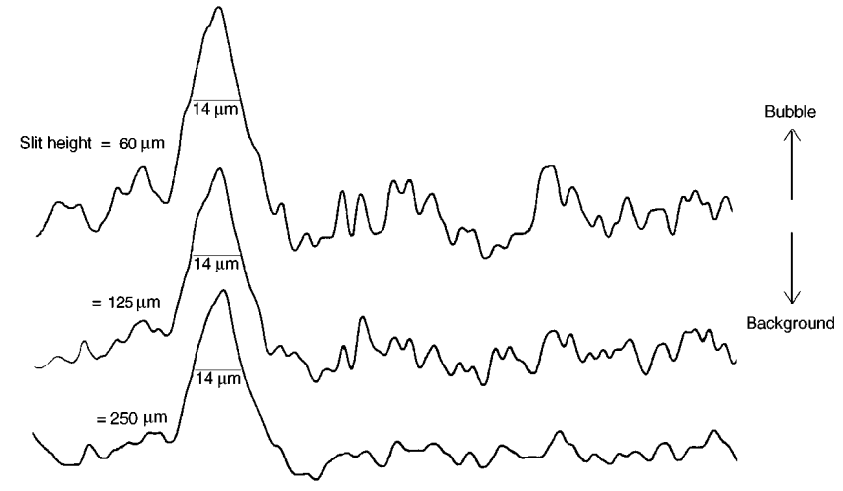

b)

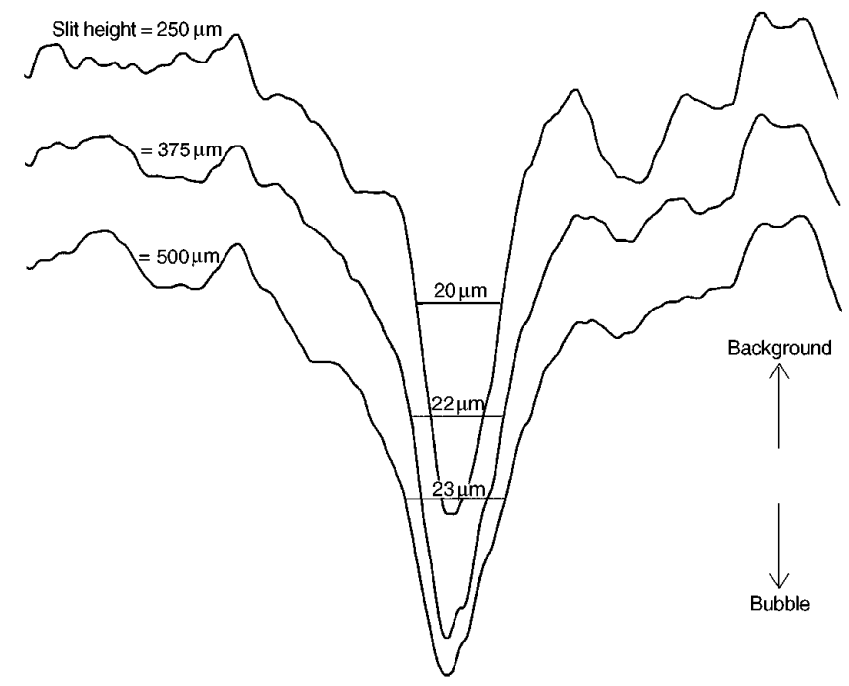

c)

Figure 14: Measurements with microdensitometer on inner branch of Vee particle (pion). Half-widths given in the curves are those measured on the film. Slit parallel to the track, slit width $20 \mu \mathrm{m}$. Density wedge: $0.056 \mathrm{D} / \mathrm{cm}$. Event time $=$ laser time $=1.664 \mathrm{~ms}$.

(a) Conventional camera, view \#6. Slit height: upper trace $500 \mu \mathrm{m}$, lower trace $250 \mu \mathrm{m}$; (b) high-resolution camera. Slit height: upper trace $60 \mu \mathrm{m}$, middle trace $125 \mu \mathrm{m}$, lower trace $250 \mu \mathrm{m}$; (c) hologram, Fig. 12. Slit height: upper trace $250 \mu \mathrm{m}$, middle trace $375 \mu \mathrm{m}$, lower trace $500 \mu \mathrm{m}$. 

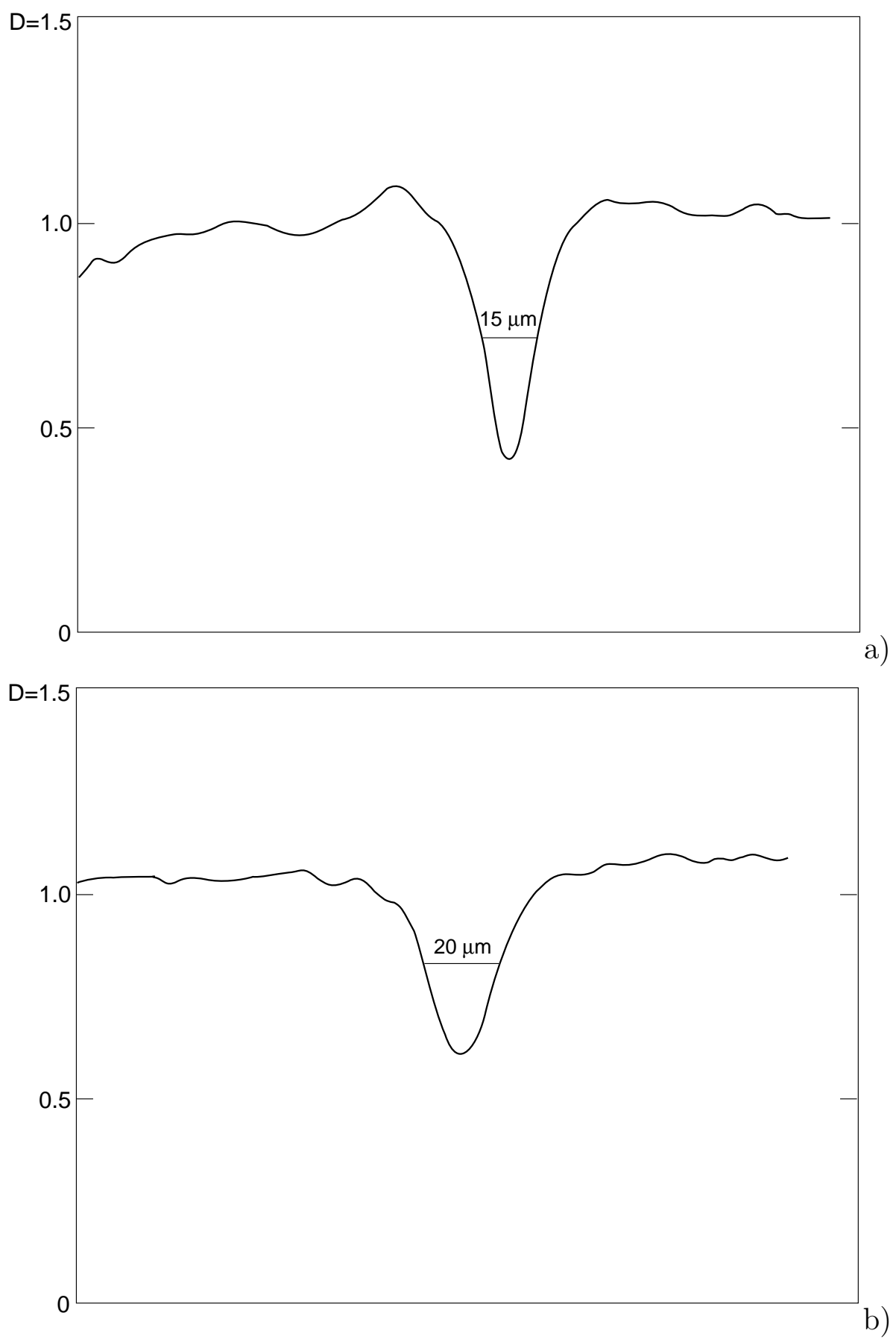

Figure 15: Measurement on high-energy muon from primary vertex far downstream. (a) conventional camera, view \#6; (b) high-resolution camera. 


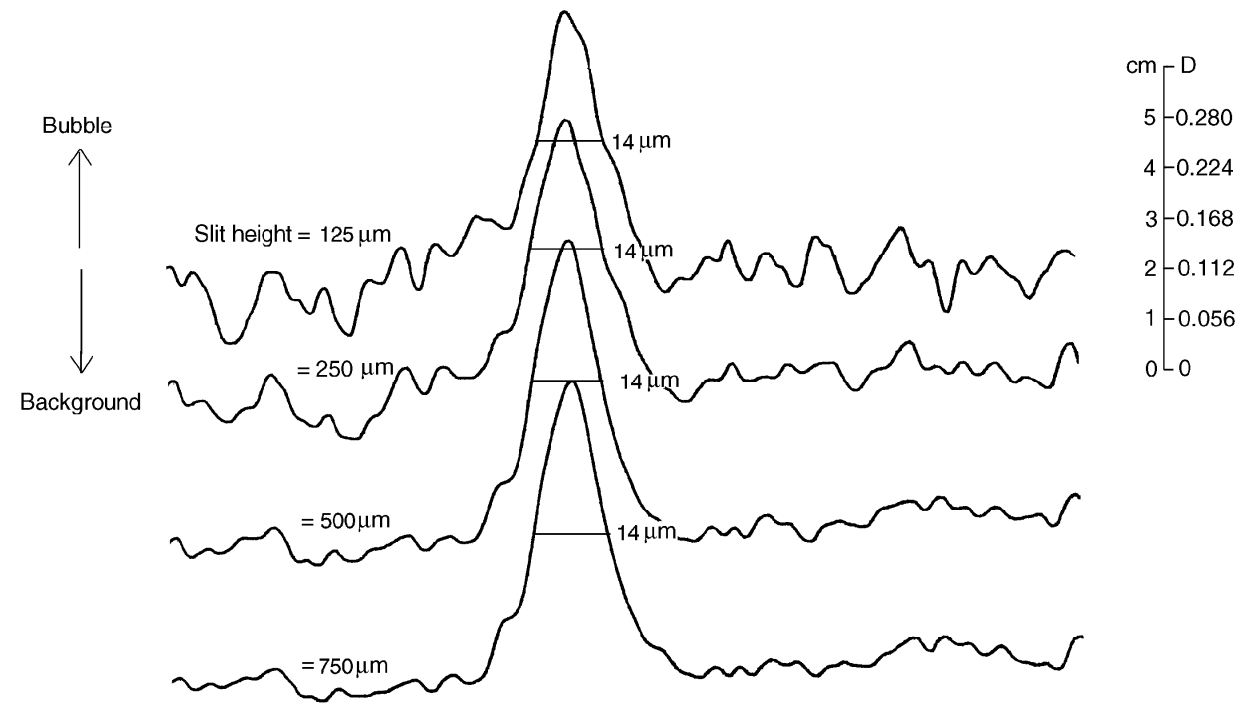

a)

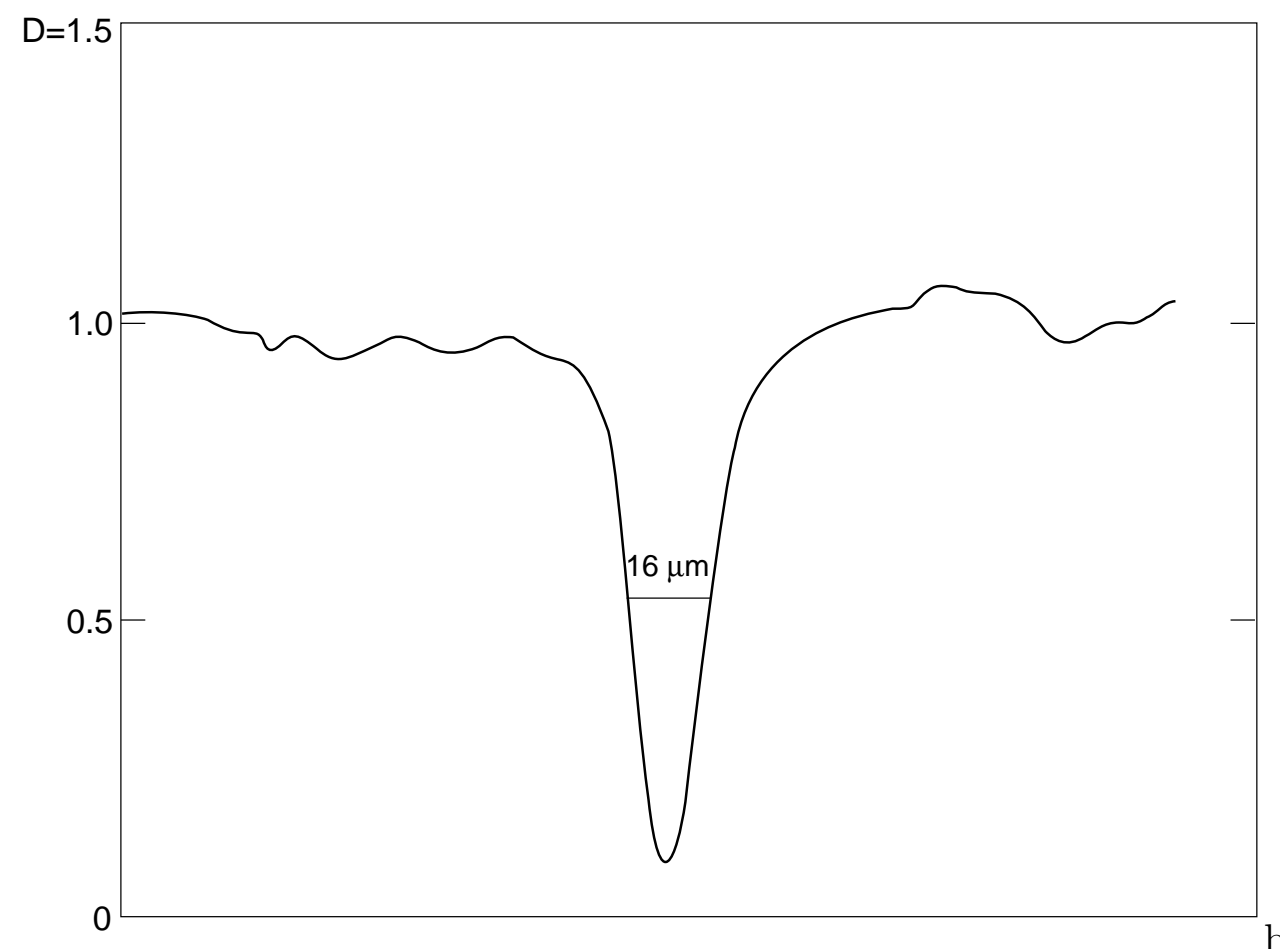

b)

Figure 16: Measurement with microdensitometer on film, slit parallel to track, on 'early' muon that occurred $1.089 \mathrm{~ms}$ before the event, beam track time $0.988 \mathrm{~ms}$ (coordinates at beginning $x=-161 \mathrm{~cm}, y=38 \mathrm{~cm}, z=+26 \mathrm{~cm}$, at end of track $x=175 \mathrm{~cm}, y=21 \mathrm{~cm}$, $z=+24 \mathrm{~cm}$ ). (a) High-resolution camera: far downstream. Slit height from top to bottom 125, 250, 500, $750 \mu \mathrm{m}$; (b) conventional camera. 


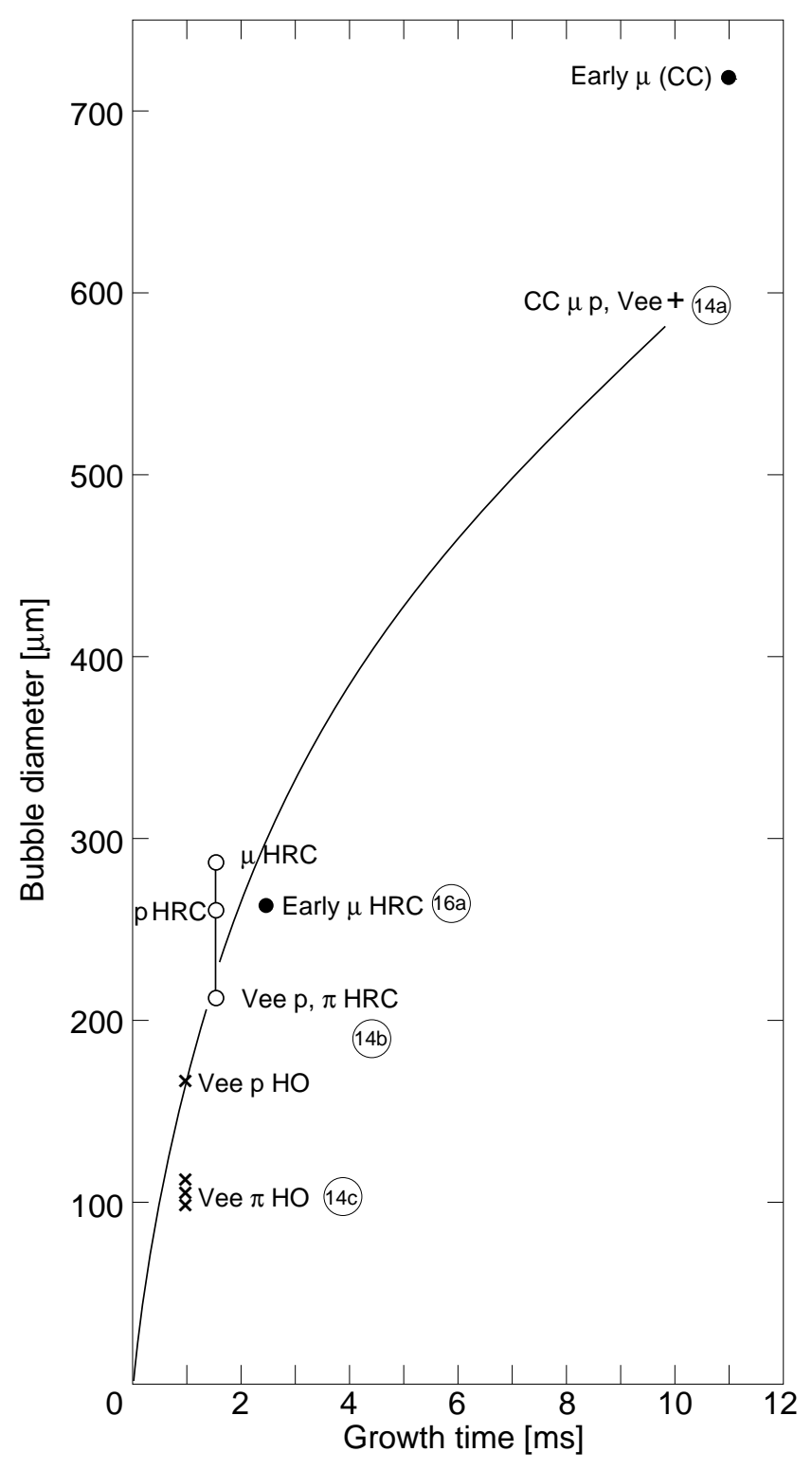

Figure 17: Summary of results of measured track width, plotted versus growth time [15][17]. HO: hologram, HRC: high-resolution camera, CC: conventional camera.

Mainly for the purpose of optic calibration, two wires (300 and $500 \mu \mathrm{m}$ thick) were installed horizontally in the mid-plane of the bubble chamber. They were also used for comparison with bubble size during adjustment of the thermodynamic chamber conditions. They were measured in the $\mathrm{CC}$ and $\mathrm{HRC}$ views (Table 4). Any comparison of wires with track measurements must take into consideration that they are non-transparent objects and that they also reflect light. 


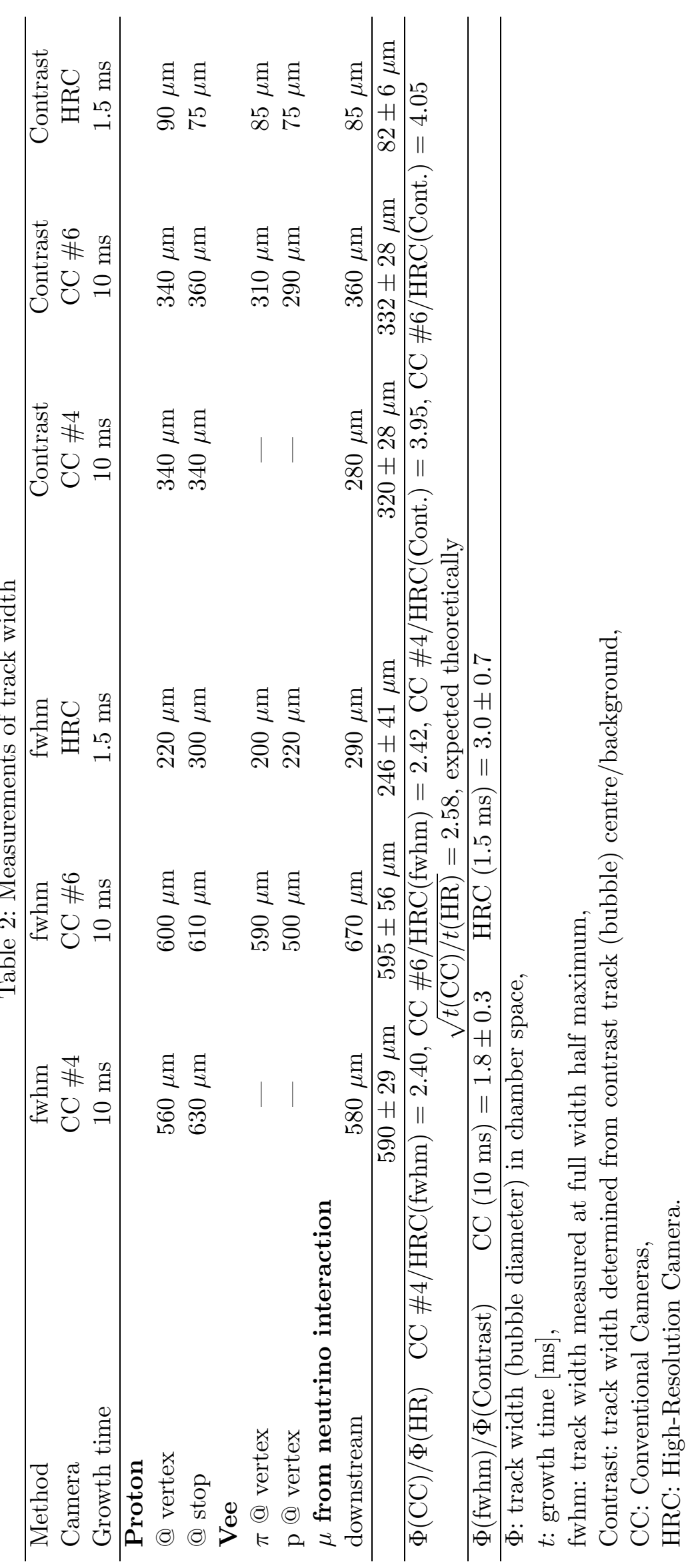




\subsubsection{Bubble size from contrast}

As has been shown in the 12-Foot Bubble Chamber at ANL, bubble sizes can be estimated by measuring the ratio of the light intensity in the centre of the aerial image of a bubble divided by the intensity in the nearby background $[38,39]$. This may be used as a measure of contrast in the aerial image; it will be denoted by $K_{\mathrm{a}}$. The corresponding ratio of transmissions of the developed emulsion is a measure of contrast in the photographic image and will be denoted by $K_{\mathrm{p}}$; this quantity is related to $K_{\mathrm{a}}$ through the characteristic curve of the emulsion.

For unresolved bubbles in bright-field illumination with Scotchlite as a retroreflector, the aerial image contrast $K_{\mathrm{a}}$ is equal to $1-D$, where $D$ is the intensity in the centre of the image of an incoherently illuminated bright disc; this has been calculated as a function of defocus, disc diameter, and lens aperture. The authors of [38] introduced three quantities:

$$
u=\frac{2 \pi n a^{2}}{\lambda}(1 / \ell-1 / R)
$$

as a measure of defocus,

$$
v=\frac{2 \pi n a \cdot \eta}{(\lambda \ell)}
$$

as a dimensionless radial co-ordinate for distance $\eta$ in the transverse plane containing the bubble, and

$$
v_{0}=\frac{2 \pi n a \cdot r}{(\lambda \ell)}
$$

the radius of the bubble in terms of $v$ units.

In these equations $2 a$ is the entrance pupil diameter of the camera lens as seen from the chamber liquid, $2 r$ the bubble diameter, $\ell$ the distance of the bubble from the pupil, $R$ the in-focus distance from the pupil and $n$ the refractive index of the liquid. These definitions are illustrated in Fig. 18.

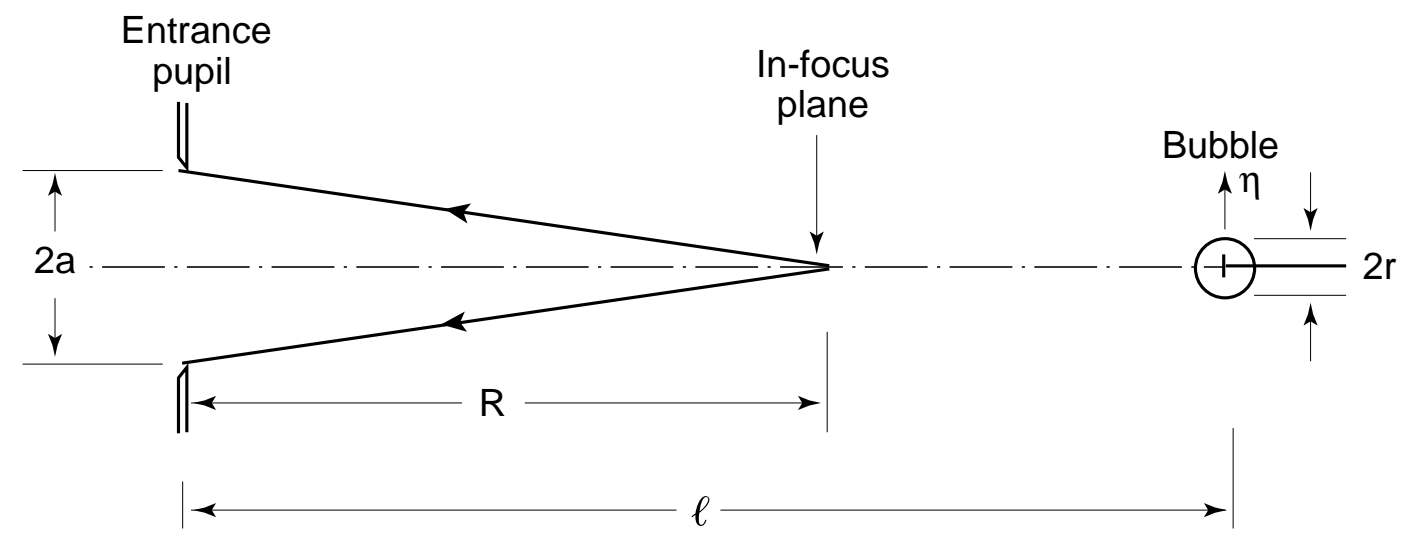

Figure 18: Illustrating the symbols used; the entrance pupil as seen from the chamber liquid through the windows.

The authors of [38] plotted $K_{\mathrm{a}}$, the contrast or central intensity, against $v_{0}$, the bubble radius, and the result is shown in Fig. 19. If the contrast, $K_{\mathrm{a}}$, and the defocus, $u$, are known, $v_{0}$ can be found. The contrast $K_{\mathrm{a}}$ is found from microdensitometer measurements on the film, yielding $K_{\mathrm{p}}$, and from the characteristic curve of the Kodak 2482 RAR film used in our experiment. This is shown in Fig. 20. 
The method is then to be applied as follows: The transmissions $T_{1}$ and $T_{2}$ of the film in the centre of the bubble image and in the bright-field background should be measured, respectively. These are then plotted on the ordinate scale of Fig. 20. These points correspond to relative exposures $E_{1}$ and $E_{2}$ on the abscissa scale, and the contrast $K_{\mathrm{a}}$ is simply $E_{1} / E_{2}$. This value is then used in Fig. 19 to find the scaled radius $v_{0}$ of the bubble, choosing the appropriate defocus $u$, and the actual bubble radius is calculated from $v_{0}$ by means of formula $(7)$.

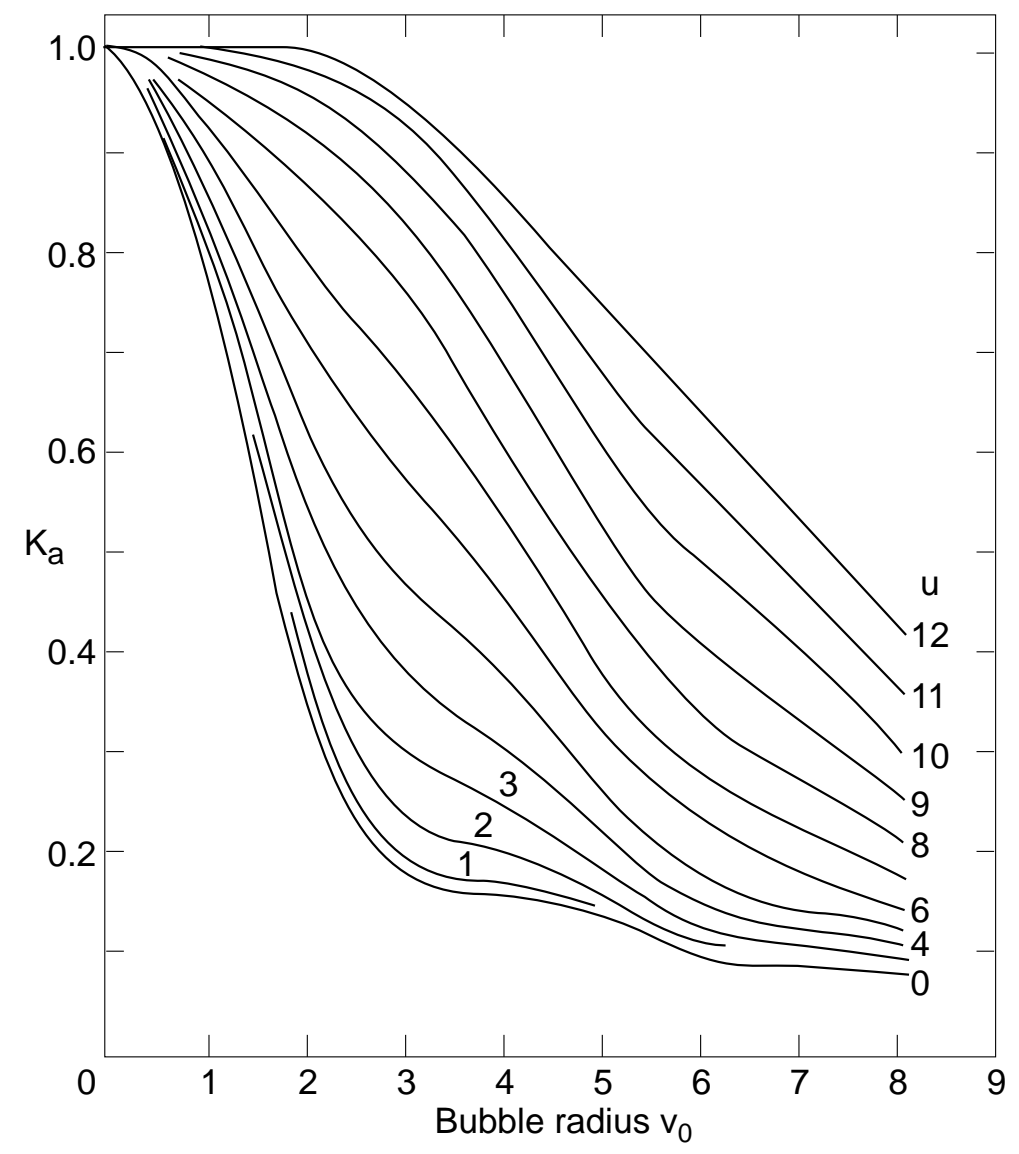

Figure 19: Contrast or central intensity $K_{\mathrm{a}}$ as a function of the rescaled bubble radius $v_{0}$, for a range of values of the defocus parameter $u$.

The application of this method to our experiment encounters two difficulties. Firstly, our densitometer has no circular spot for the measurement, and, secondly, the bubble density is so high that individual bubbles can not be identified reliably. In order to get reasonable information of the transparency of the background in the vicinity of the bubbles we had to rely on the measurements with the slit moving parallel to the track over the bubble string.

Our microdensitometer measures optical densities, $D$, which have to be transformed into transparencies, $T$, by using the relation

$$
D=\log _{10}\left(\frac{1}{T}\right)
$$

The density wedges we used were $0.056 \mathrm{D} / \mathrm{cm}$ and $0.084 \mathrm{D} / \mathrm{cm}$ on the paper recording. The absolute calibration was done with a $1.5 \mathrm{D}$ filter in the object space. An additional 
check of the transmission $\mathrm{T}$ was done without the film in place (perfect transmission, $T=1$ ), then at a region of the developed film outside the bubble chamber image (overall fog density of the developed emulsion support, $T=0.97)$. These values gave the baseline for the measurements of the background near the track, and in the centre of the track, each for identical settings of slit height and width.

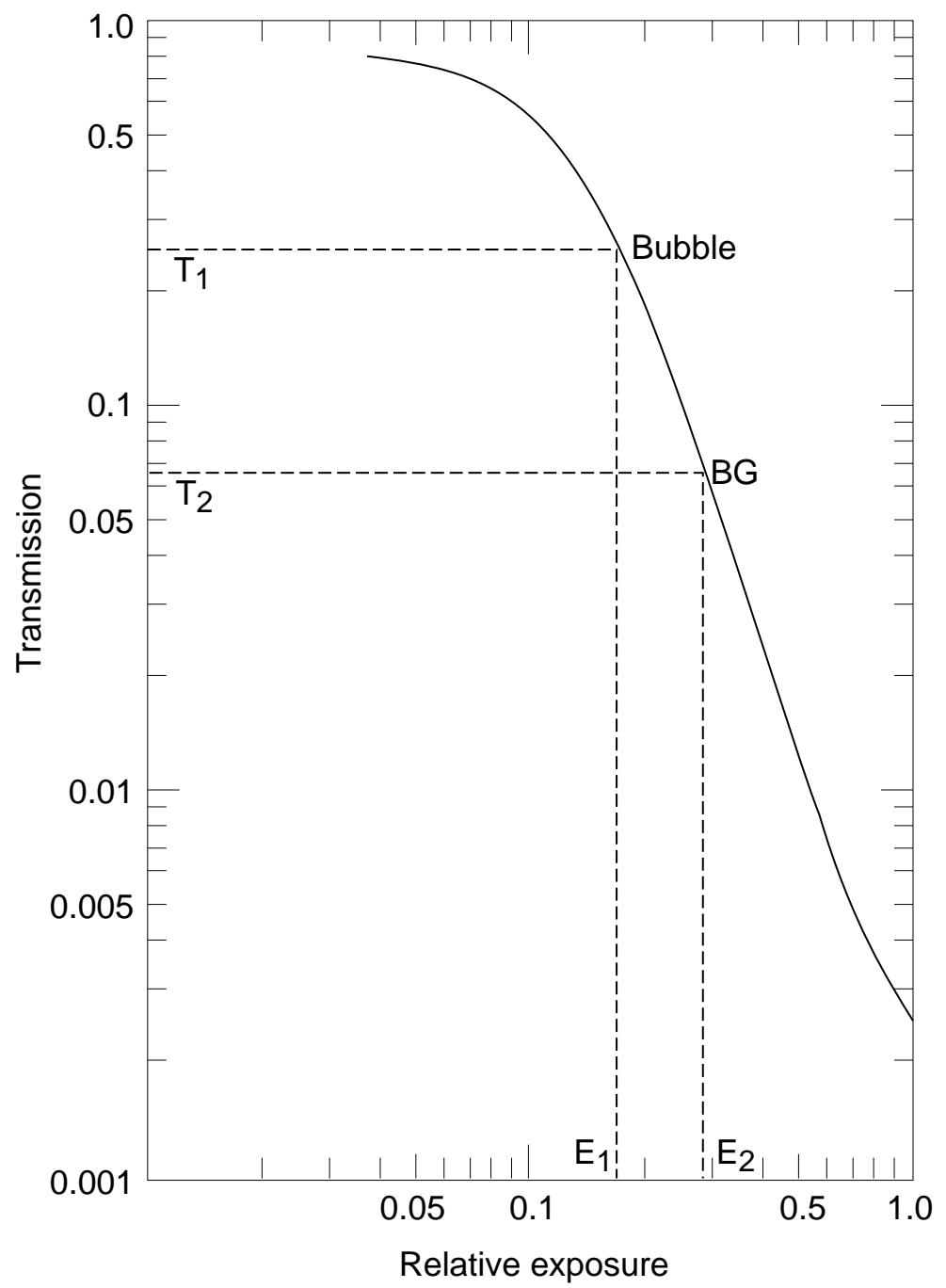

Figure 20: The characteristic curve, transmission-exposure, for Kodak 2482 RAR emulsion: exposure $10^{-4} \mathrm{~s}$, developed $8 \mathrm{~min}$ at $20^{\circ} \mathrm{C}$ in D19 developer. Data by courtesy of Argonne National Laboratory and Prof. E. Wilson Powell.

Results of the contrast method are given in Tables 2 and 3 (last 3 columns) for the tracks at various growth times for the $\mathrm{CC}$ and $\mathrm{HCR}$ views. There is a remarkable factor of 1.8 (CC) and 3.0 (HRC) in the size using the fwhm and contrast methods. However, the range of these factors is very similar to the ones found in [38]. Since the smaller sizes were in better agreement with experiments done with $1: 1$ optics in the DESY bubble chamber $[34,35]$, we have confidence that the presented contrast method gives the more reliable data. The results are plotted in Fig. 21, showing three square-root time dependencies for curves fitted through the measured values. There is no explanation for the difference between the curves fitted through short and long time delays, since owing to the well- 
established $\sqrt{t}$-dependence all measured diameters should fall on one curve. These curves indicate that the bubble size, when the hologram is taken, can be expected to be between 60 and $110 \mu \mathrm{m}$. There is a fairly good agreement of the time dependence in the HRC for $1.5 \mathrm{~ms}$ and $2.5 \mathrm{~ms}$ exposures, and in the CC between $10 \mathrm{~ms}$ and $11 \mathrm{~ms}$ exposures, but the difference between the two camera sets is important. The width of the wires were also measured by the contrast method (which is not derived for solid objects) and are shown in Table 4.

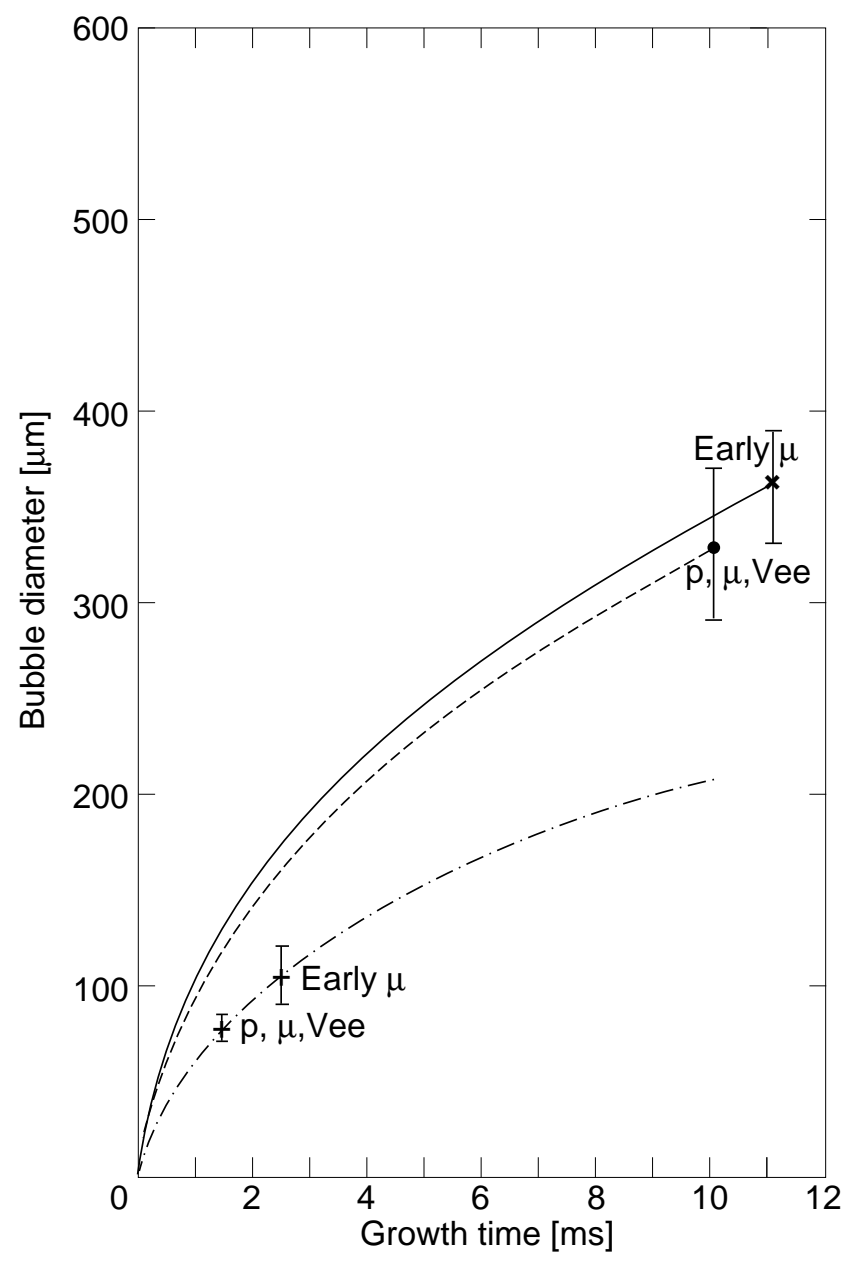

Figure 21: Bubble diameter as function of growth time, using the contrast method [36, 38]. Calculated square-root of time dependence, using data from early muon (11 ms), solid line; $\mathrm{p}, \mu$, Vee (10 ms), dashed line; early muon (2.5 ms) and p, $\mu$, Vee $(1.5 \mathrm{~ms})$, dashed-dotted line.

Table 4: Measurements of horizontal wires ${ }^{\text {a) }}$

\begin{tabular}{|c|c|c|c|c|c|c|}
\hline Method & fwhm & fwhm & Contrast & Contrast & $\begin{array}{c}\Phi(\text { fwhm }) / \\
\Phi(\text { Cont. })\end{array}$ & $\begin{array}{r}\Phi(\text { fwhm }) / \\
\Phi(\text { Cont. })\end{array}$ \\
\hline Camera & $\mathrm{CC} \# 4$ & HRC & $\mathrm{CC} \# 4$ & HRC & $\mathrm{CC} \# 4$ & HRC \\
\hline Thickness & & & & & & \\
\hline $300 \mu \mathrm{m}$ & $540 \mu \mathrm{m}$ & $360 \mu \mathrm{m}$ & $190 \mu \mathrm{m}$ & $160 \mu \mathrm{m}$ & 2.84 & 2.25 \\
\hline $500 \mu \mathrm{m}$ & $670 \mu \mathrm{m}$ & $520 \mu \mathrm{m}$ & $360 \mu \mathrm{m}$ & $170 \mu \mathrm{m}$ & 1.86 & 3.06 \\
\hline
\end{tabular}

$\Phi$ : Wire width in space $[\mu \mathrm{m}]$.

a) $x$-co-ordinate of measurements near event vertex. 


\subsubsection{Bubble size from bubble density}

The measured bubble density along a track depends upon the time of photography; it decreases with increasing flash delay $t(\mathrm{~s})$. It can be reasonably assumed that coalescence of bubbles is responsible for this decrease (compare Fig. 10). A simple relation between primary bubble density $N_{0}$ and the bubble radius $r(\mathrm{~cm})$ exists and had been experimentally verified [36] (Fig. 22). We chose in analogy with counting losses in counter experiments the relation

$$
N=\frac{N_{0}}{\left(1+2 N_{0} r\right)},
$$

where $N$ is the number of bubbles/cm at a given time $t$ (s) (the moment of photography) and $N_{0}$ is the number of bubbles originally produced. The bubble diameter grows with

$$
r[\mathrm{~cm}]=A\left[\mathrm{~cm} / \mathrm{s}^{-1 / 2}\right] \sqrt{t}[\mathrm{~s}] .
$$

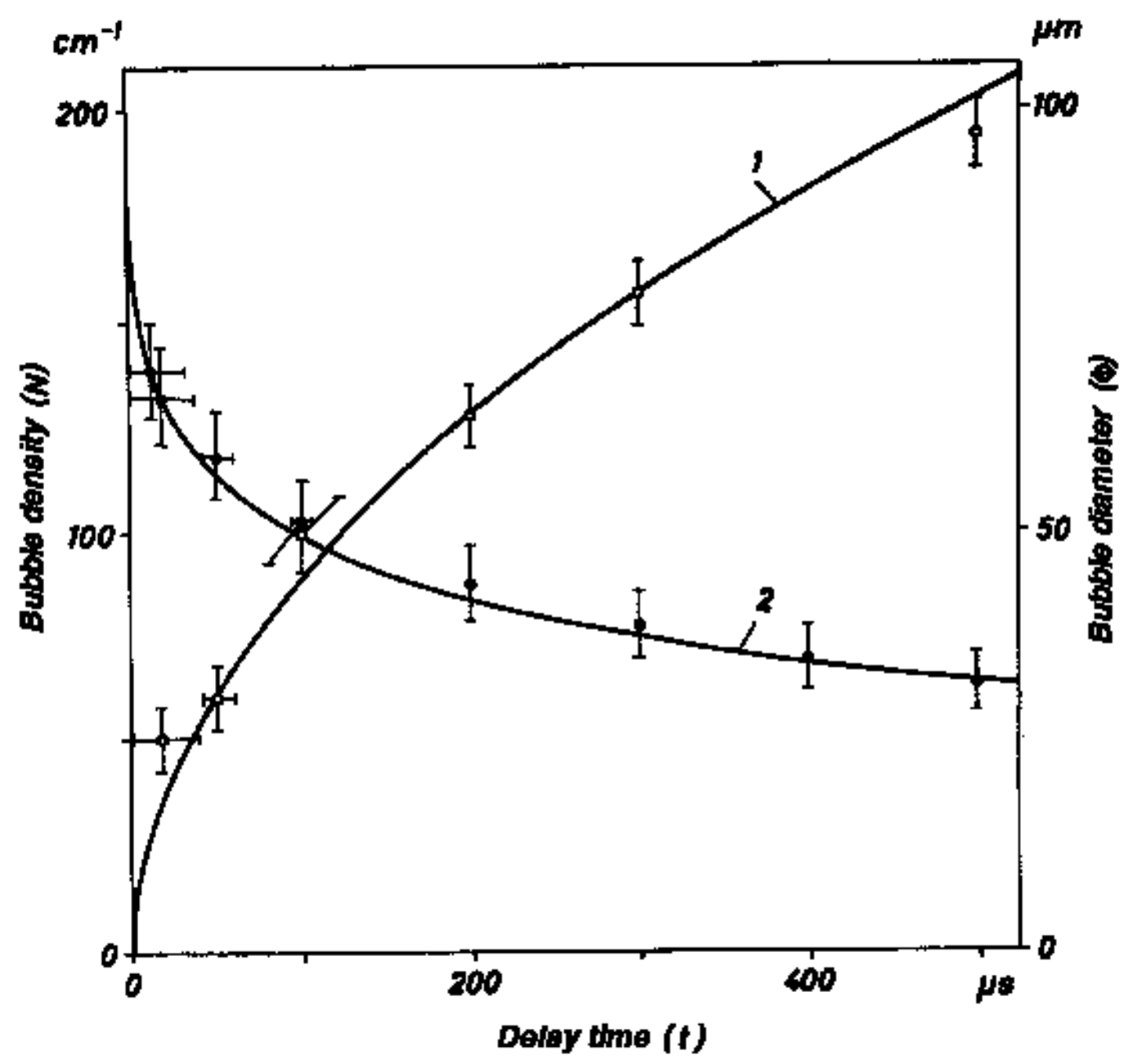

Figure 22: Results from an unpublished propane bubble chamber experiment [36]. Measured bubble diameters (curve 1) and (curve 2) densities as a function of time delay. They are fitted by best curves, in agreement with Eq. (9). Extrapolation to delay time zero resulted in 180 bubbles/cm. 
From an experiment performed in the DESY $85-\mathrm{cm}$ bubble chamber with a $1: 1$ magnification optic we know the value of the growth factor $A$ for two neon/hydrogen mixtures, one with a significantly higher and one with a somewhat lower neon concentration than the mixture ratio used in the present experiment [34] (Fig. 24). Furthermore, we show the bubble densities [34] (Fig. 23), extrapolated to the injection time, for these two mixtures as a function of expanded pressure. The value of the expanded pressure in the 15-Foot Chamber is not known owing to the absence of an absolute calibration of the dynamic pressure transducers. We make the assumption that at least the same low minimum pressure was obtained as in the DESY chamber. The justification lies in the fact that the smaller surface-to-volume ratio in larger chambers makes it possible to reach lower expanded pressures due to less parasitic boiling. Consequently, a density $N_{0} \cong 100$ bubbles/cm could be assumed for our experiment in the 15-Foot Chamber.

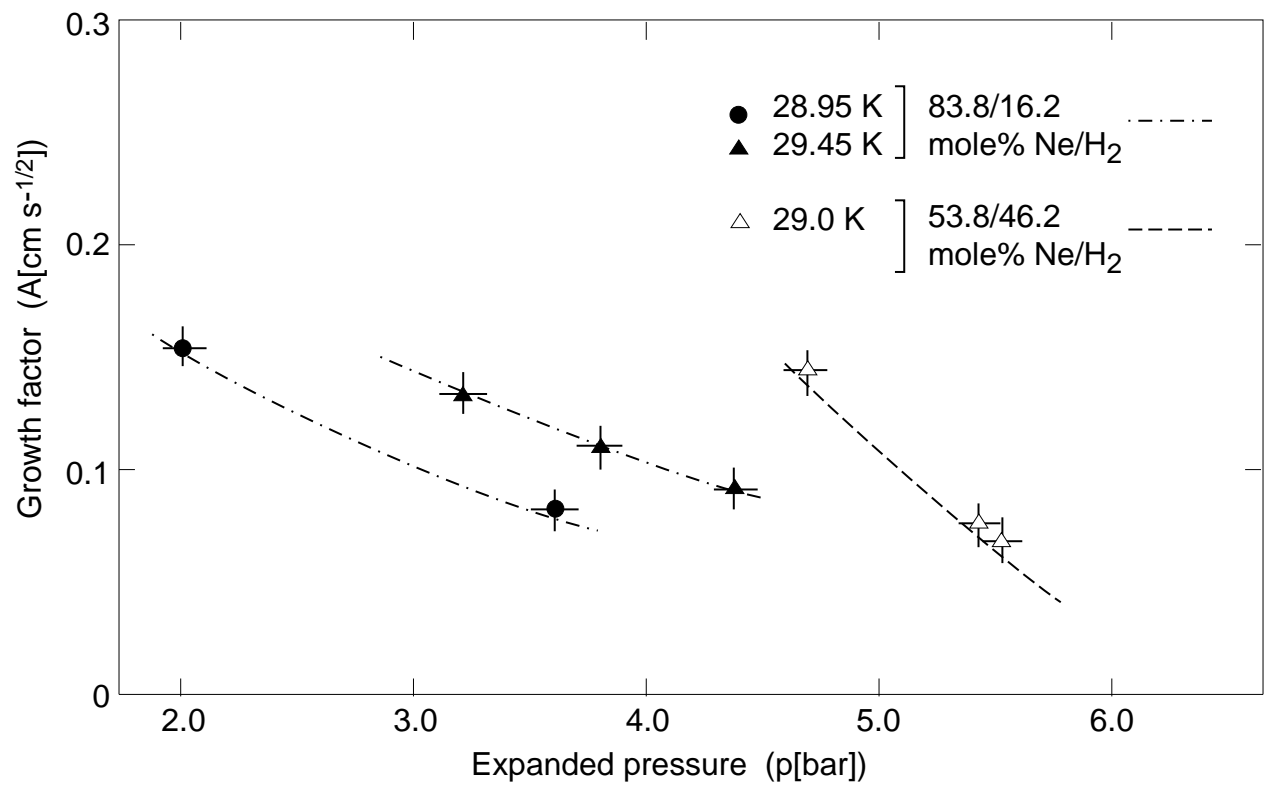

Figure 23: The measured growth factor $A$ for bubbles, as defined in the $(d=2 A \sqrt{t})$ law, as a function of pressure for two $\mathrm{Ne} / \mathrm{H}_{2}$ mixtures, taken from [34]. 


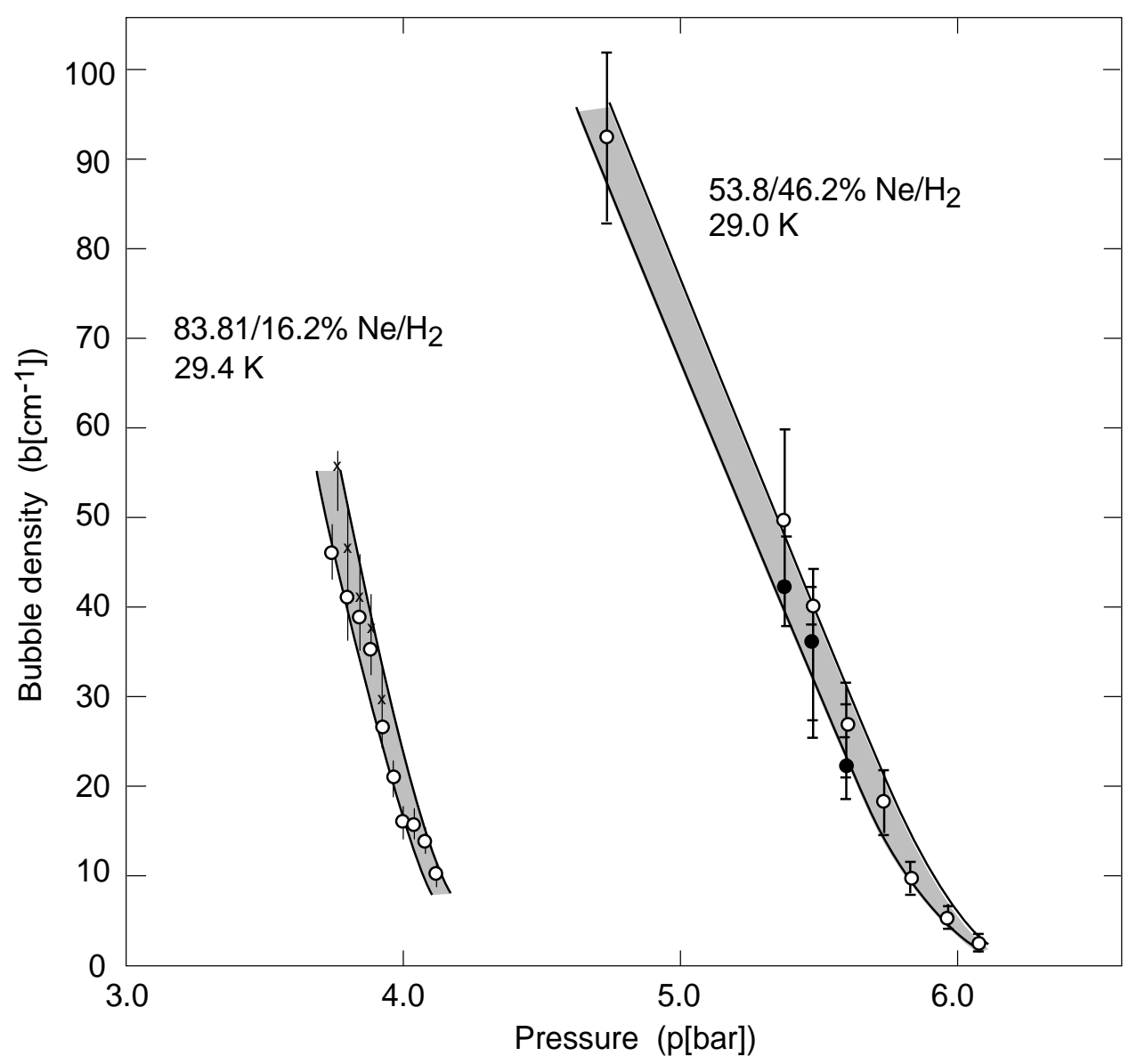

Figure 24: Bubble density as a function of pressure for two $\mathrm{Ne} / \mathrm{H}_{2}$ mixtures, taken from [34]. 
As is evident from the hologram [Fig. 11(c)], the fuzziness of the tracks does not allow (reliable) bubble density measurements. We were limited in this investigation to the two bright-field recordings. On the high-resolution film we found on the outer branch of the Vee particle (proton) a bubble density of 52 bubbles/cm at a delay of $t=1.5 \mathrm{~ms}$, and on the early muon a density of 42 bubbles/cm at the delay of $2.5 \mathrm{~ms}$. On the conventional film we measured on the primary muon far downstream 17 bubbles $/ \mathrm{cm}$ at $t=10 \mathrm{~ms}$ (all numbers are in bubble chamber space). Figures 25, 26 and 27 show the corresponding measured variation of the optical density when moving along the track with the microdensitometer slit perpendicular to the track. It is obvious from these figures that there are large uncertainties in defining the image of a bubble. The definition of the background level remains somewhat arbitrary due to inhomogeneity of the Scotchlite reflection. Furthermore, if we apply strictly the Rayleigh criterion for the resolution of objects we have to count overlapping images as separate bubbles. Using the latter definition we find for the cases discussed above 70, 50, and $22 \mathrm{~b} / \mathrm{cm}$, respectively.

The bubble density at the time of the passage of the particles (time zero) must have been the same, with the exception of the difference between their ionizing power, which we neglect in the present context. Assuming $N_{0} \cong 100$ bubbles $/ \mathrm{cm}$, we use formula (9) and the measured bubble densities to determine the bubble diameters $2 r$. We find the values given in Table 5. The calculated diameters are plotted in Fig. 28. 


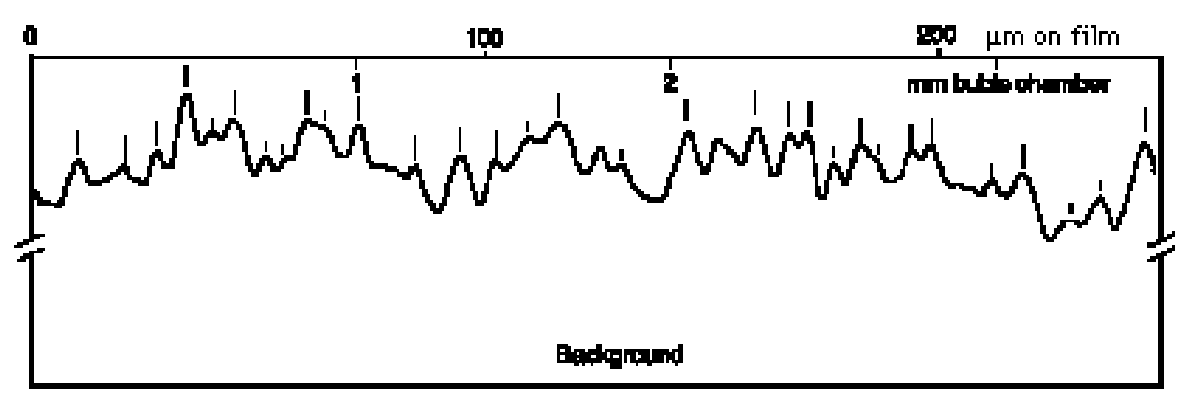

Figure 25: Measurement with microdensitometer on outer branch of Vee particle (proton). Slit perpendicular to the track, slit width $30 \mu \mathrm{m}$. Density wedge: $0.056 \mathrm{D} / \mathrm{cm}$. Event time $=$ laser time $=1.664 \mathrm{~ms}$. Chamber co-ordinates of origin of Vee: $x=18 \mathrm{~cm}, y=-11 \mathrm{~cm}$, $z=9 \mathrm{~cm}$. High-resolution camera. Slit height: $1 \mathrm{~mm}$. Maxima assumed to be centre of bubble image: long vertical marks: very probable, short vertical marks: less probable.

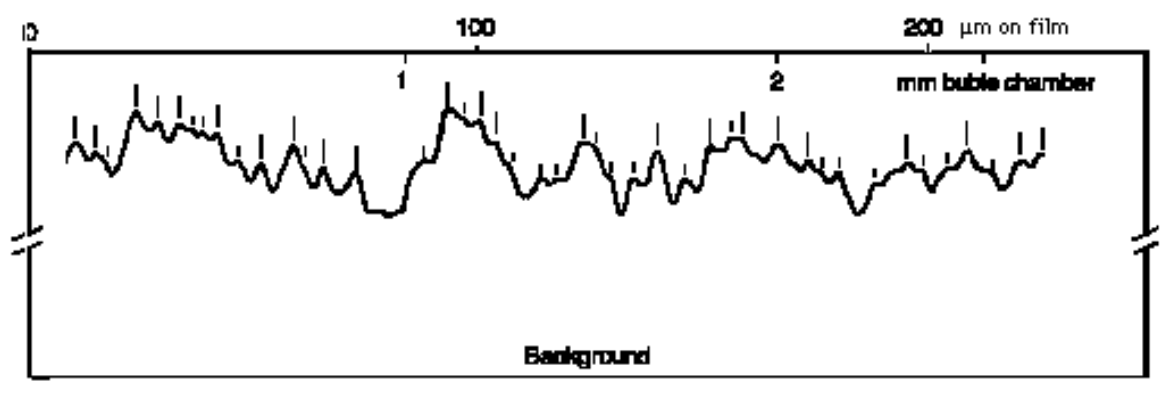

Figure 26: Measurement with microdensitometer on early muon. Slit perpendicular to the track, slit width $20 \mu \mathrm{m}$. Density wedge: $0.056 \mathrm{D} / \mathrm{cm}$. $1.089 \mathrm{~ms}$ before event. Chamber co-ordinates: $x=175 \mathrm{~cm}, y=-21 \mathrm{~cm}, z=24 \mathrm{~cm}$. High-resolution camera. Slit height: $200 \mu \mathrm{m}$.

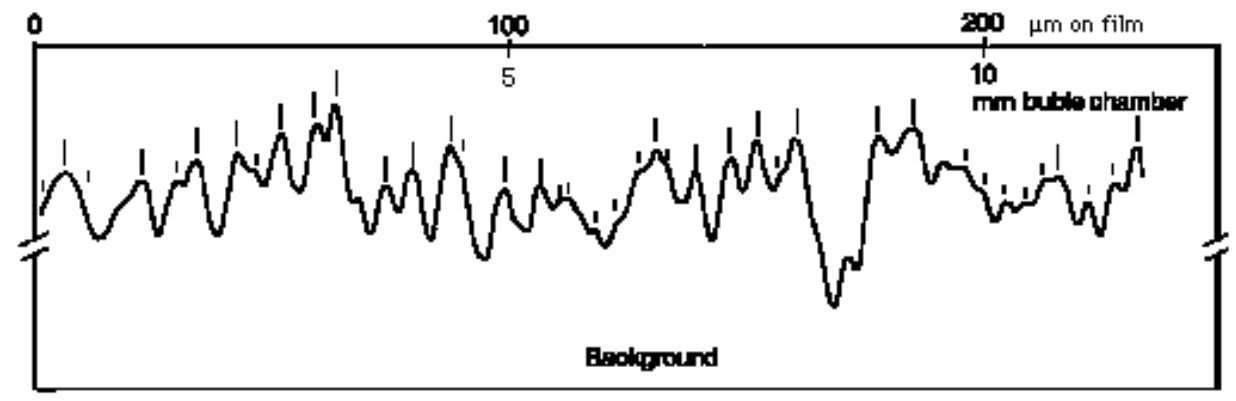

Figure 27: Measurement with microdensitometer on muon from primary vertex far downstream. Slit perpendicular to the track, slit width $20 \mu \mathrm{m}$. Density wedge: $0.056 \mathrm{D} / \mathrm{cm}$. Event time $=1.664 \mathrm{~ms}$. Chamber co-ordinates at exit point: $x=170 \mathrm{~cm}, y=-17 \mathrm{~cm}$, $z=32 \mathrm{~cm}$. Conventional camera, view 6. Slit height: $75 \mu \mathrm{m}$. 


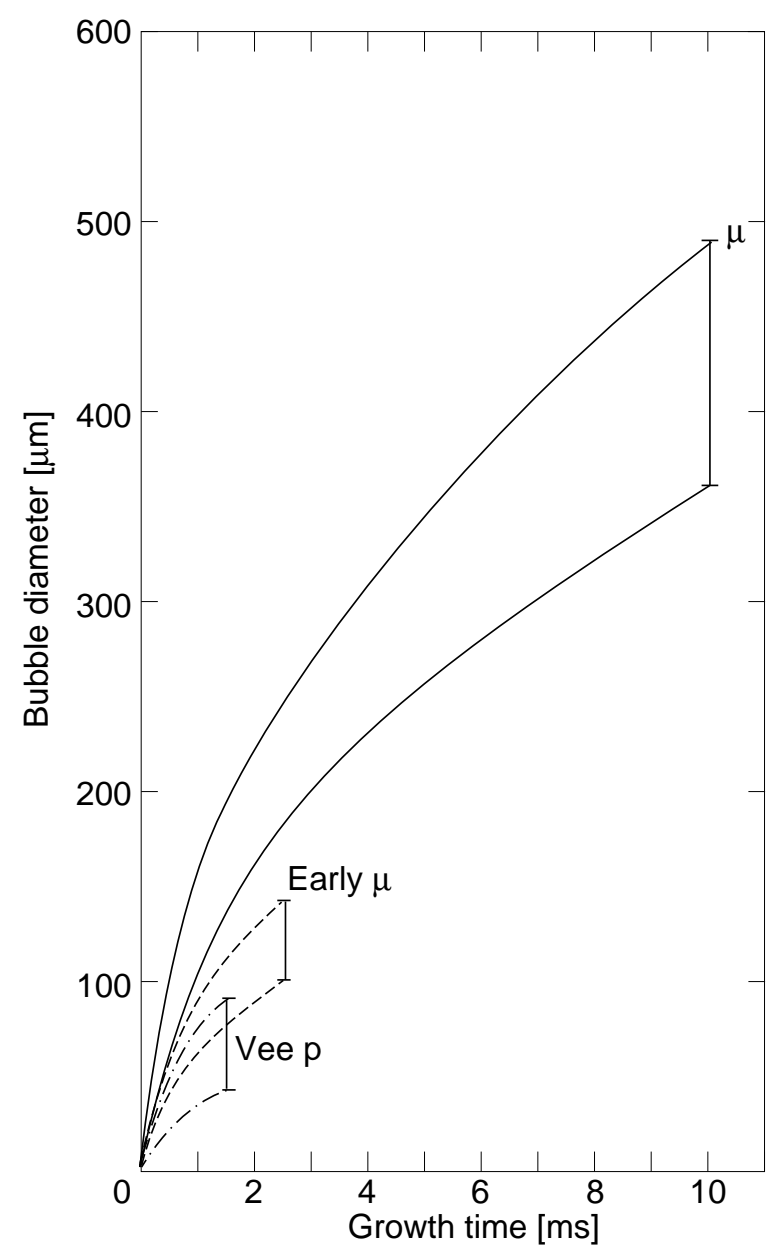

Figure 28: Bubble diameters as function of growth time, using bubble density method [36]. The range of bubble diameters for each point results from applying or not the strict Rayleigh criterion for the resolution of overlapping objects. Calculated square-root of time dependence, using $\mu$ (10 ms), solid line; early $\mu(2.5 \mathrm{~ms})$, dashed line; Vee p (1.5 ms), dashed-dotted line.

The values of $A$, evaluated from formula (10), using these calculated bubble diameters are within the range covered by the DESY experiment for both the minimum and maximum number of bubbles counted (see Fig. 24). The limits of this numerical procedure are evident from the scattering of the $A$ values, which should be identical in order to satisfy the $\sqrt{t}$-law. The deviation can be explained partially by the variation of the expanded pressure during the growth of the bubbles, which has a larger effect for longer growth periods. The calculated values are consistently lower than the measured values of the bubble diameters. Reasons for this are:

1) our two optics arrangements have diffraction-limited resolutions of $200 \mu \mathrm{m}$ and $500 \mu \mathrm{m}$, respectively, and

2) as shown earlier, we measure systematically larger images due to the properties of our measuring apparatus, due to the presence of small $\delta$-electrons, and due to the jitters of bubbles around the trajectory of the particles.

Within the given precision, taking the average of the $A$ values, we find with our method a bubble size of 107 (70 with strict Rayleigh criterion) $\mu \mathrm{m}$ for the hologram taken 
with $1 \mathrm{~ms}$ delay, albeit with large uncertainty. Figure 28 shows a range of 70 to 155 (30 to 100$) \mu \mathrm{m}$ at $1 \mathrm{~ms}$.

Table 5: Measured and calculated bubble diameters using the bubble density method. Values in parentheses result from the strict application of the Rayleigh criterion for the resolution of overlapping objects

\begin{tabular}{|c|c|c|c|c|}
\hline $\begin{array}{l}\text { Photo, delay, } \\
\text { particle }\end{array}$ & $\begin{array}{l}N\left(\mu \mathrm{m}^{-1}\right) \\
\text { Measured }\end{array}$ & $\begin{array}{l}2 r(\mu \mathrm{m}) \\
\text { Measured } \\
\text { fwhm-method }\end{array}$ & $\begin{array}{l}2 r(\mu \mathrm{m}) \\
\text { Calculated } \\
\text { bubble density } \\
\text { method }\end{array}$ & $\begin{array}{l}A\left(\mathrm{~cm} \mathrm{~s}^{-1 / 2}\right) \\
\text { Calculated }\end{array}$ \\
\hline $\begin{array}{l}\text { High-resolution, } 2.5 \mathrm{~ms} \text {, } \\
\text { 'early' muon }\end{array}$ & $\begin{array}{c}42 \cdot 10^{-4} \\
\left(50 \cdot 10^{-4}\right)\end{array}$ & 260 & $\begin{array}{l}140 \\
(100)\end{array}$ & $\begin{array}{l}0.15 \\
(0.10)\end{array}$ \\
\hline $\begin{array}{l}\text { High-resolution, } 1.5 \mathrm{~ms} \text {, } \\
\text { proton from Vee }\end{array}$ & $\begin{array}{c}52 \cdot 10^{-4} \\
\left(70 \cdot 10^{-4}\right)\end{array}$ & 210 & $\begin{array}{l}90 \\
(40)\end{array}$ & $\begin{array}{l}0.12 \\
(0.06)\end{array}$ \\
\hline $\begin{array}{l}\text { Hologram, } 1.0 \mathrm{~ms} \text {, } \\
\text { pion from Vee }\end{array}$ & & 100 & & \\
\hline $\begin{array}{l}\text { Convent. camera, } 10 \mathrm{~ms} \text {, } \\
\text { primary muon }\end{array}$ & $\begin{array}{c}17 \cdot 10^{-4} \\
\left(22 \cdot 10^{-4}\right)\end{array}$ & 600 & $\begin{array}{l}490 \\
(350)\end{array}$ & $\begin{array}{l}0.24 \\
(0.18)\end{array}$ \\
\hline
\end{tabular}

\subsubsection{Conclusions from the track size measurements}

The main results of the measurements are shown in Figs. 13, 17, 21 and 28, where only statistical errors were given. Systematic errors arise from the measuring technique, such as the slightly imperfect parallel alignment of the slit and the track segment, partially due to the curvature of the tracks, small $\delta$-electrons, jitters of bubbles along the particle trajectory, and the illumination angle. Superimposed is the effect of defocus, caused when tracks have large dip angles or measured track segments are far away from the event vertex. No attempt was made to correct for defocus. The overall systematic error is estimated to be about $\pm 10 \%$.

The fwhm method overestimates the bubble size, but gives fairly consistent results over the large growth interval, i.e. the measured points are all close to one $\sqrt{t}$-line. The contrast method gives consistently smaller bubble sizes, but shows substantial differences in the growth factor $A$ (formula 10) between short and long times. The calculations from bubble densities give values of sizes in between the two methods described above, albeit with considerable spread. Taking the results of the three methods together one can conclude that the diameter of bubbles at $1 \mathrm{~ms}$, the time when the hologram is taken, is probably $\leq 120 \mu \mathrm{m}$.

Direct measurements of the fuzzy track images in holographic replay, mainly due to the speckle, have only limited value for our analysis of the beam branching ratio. However, the uncertainty in the determination of track width does not diminish the usefulness of holograms, since they allow for a good visual inspection of event vertices, of possible kinks along a track, and for identification of secondary vertices within a volume much larger than obtainable with high-resolution optics. 
The resolution of the holographic images is affected by a combination of aberrations originating from the recording and reconstruction processes. Aberrations are due to the differences in wave front between the reconstructing illumination beam and the recording reference beam, and film limitations such as shrinkage and imperfections of the emulsion. Aberrations due to a difference in wave front between the illumination beam and the reference beam consist partly of geometrical aberrations introduced by the fish-eye windows. A difference in wave front between illumination and reference beam is also introduced by any source of scattering near the dispersing lens, mainly from the micro-boiling.

The resolution can only be measured for the combined holographic system. The performance of both the recording and replay devices is important. The measurement of the resolution is made during tests in the open (i.e. air-filled) bubble chamber with a 1951 USAF (United States Air Force) bar target placed at the centre of the bubble chamber approximately $2 \mathrm{~m}$ away from the holographic film plane. The smallest possible separation of lines was visually determined to be $\sim 157 \mu \mathrm{m}$ at the FNAL replay machine [26].

An interesting characteristic of the holographic system is the size of the visible volume. This depends strongly on the $B B R$ of the bubbles, and thus on their diameter or growth time. Taking the various geometric factors into account and using the predicted events from the conventional film and the events found in holograms, it was determined to be $1.4 \mathrm{~m}^{3}$.

Figure 29 shows a scatter plot of the position of the events within the $\left(z_{\mathrm{hol}}, R_{\mathrm{hol}}\right)$ plane. An important quantity in the design of the dispersing lens, and ultimately for the quality of the holograms and the size of the visible volume, is the minimum-detectable $B B R$ [Eq. (2)]. The position of the event vertex allows the calculation of the geometrical quantities $d_{\mathrm{i}}, d_{\mathrm{h}}, \alpha$ and $\Theta_{\mathrm{h}}$. Since only the events for which the bubble had a growth time of $1 \mathrm{~ms}$ are retained, the corresponding bubble diameter is estimated at a nominal value of $110 \mu \mathrm{m}$.

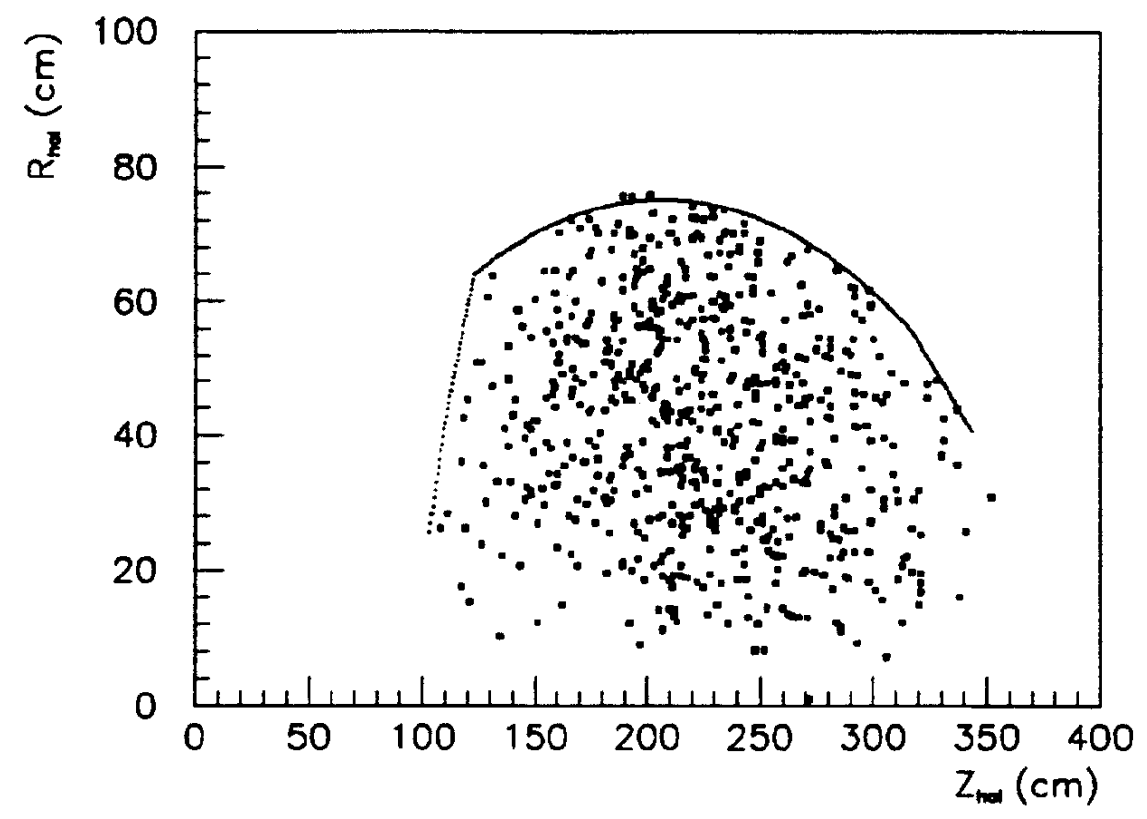

Figure 29: Scatter plot of the event vertices within the scanned holographic volume. 
The design values from $F\left(\Theta_{\mathrm{i}}\right)$ and $I_{\mathrm{r}}$ are used under the assumption of a uniform laser light intensity distribution. For the light scattering function $G(\alpha)$, the theoretical estimates are used.

Systematic uncertainties in the determination of the $B B R$ originate mainly from two sources: the non-uniformity of the laser light intensity profile at the dispersing lens and the change in bubble chamber conditions during the recording of holograms. Although the absolute incident intensity value does not affect the $B B R$, differences between the intensity at the centre of the lens, where the reference beam is generated, and at the portion generating the illumination for a bubble do change the $B B R$. The spatial variation in light intensity across the entrance at the dispersing lens can be calculated from the beam profile data stored on the EMI tapes. The variation is typically $25 \%$ around the mean value. A change in bubble chamber conditions results in a variation of the bubble radius $r$. A conservative estimate of the overall variation $\Delta r$, based on the change in expanded pressure, is $5 \mu \mathrm{m}$, i.e. 8.5\%. The total systematic error on the $B B R$ is therefore estimated at $\pm 39 \%$ of its nominal value. This range corresponds to an error of \pm 0.18 in $\log _{10} B B R$.

Table 6 displays the event reconstruction efficiency of the replay devices as a function of various ranges in $\log _{10} B B R$. The size of these intervals is determined by the systematic error on the $B B R$. In the interval of $B B R$ between $0.44 \times 10^{-6}$ to $10^{-6}$, i.e. $-6.36 \leq \log _{10} B B R \leq-6$, the event reconstruction efficiencies are approximately equal for HOLRED, the FNAL, and HAM replay machines. The reconstruction efficiency for the Hawaii device is significantly lower in this interval. This can be partly explained by the lower average exposure or underexposure of the holograms analysed with the Hawaii machine.

Table 6: Event reconstruction efficiency as a function of $B B R$ for the various replay devices

\begin{tabular}{lllllll}
\hline \multicolumn{5}{c}{ Optical reconstruction efficiencies } \\
\hline$B B R$ & $\log _{10} B B R$ & HOLRED & Hawaii & FNAL & HAM & All \\
$0.54 \cdot 10^{-7}$ & & {$[25]$} & {$[24]$} & {$[26]$} & {$[27]$} & \\
& & & & & & \\
$0.84 \cdot 10^{-7}$ & {$[-7.27,-7.07]$} & $0.33 \pm 0.05$ & $0.07 \pm 0.03$ & $0.58 \pm 0.04$ & $0.27 \pm 0.11$ & $0.35 \pm 0.03$ \\
$1.94 \cdot 10^{-7}$ & {$[-7.07,-6.71]$} & $0.56 \pm 0.04$ & $0.26 \pm 0.04$ & $0.70 \pm 0.03$ & $0.40 \pm 0.08$ & $0.53 \pm 0.02$ \\
$4.37 \cdot 10^{-7}$ & {$[-6.71,-6.36]$} & $0.71 \pm 0.05$ & $0.55 \pm 0.07$ & $0.86 \pm 0.03$ & $0.55 \pm 0.11$ & $0.73 \pm 0.03$ \\
$10 \cdot 10^{-7}$ & {$[-6.36,-6.00]$} & $0.81 \pm 0.08$ & $0.53 \pm 0.12$ & $0.89 \pm 0.06$ & $0.88 \pm 0.08$ & $0.79 \pm 0.04$ \\
\hline
\end{tabular}

For a well-tuned machine, $B B R$ down to $(0.13 \pm 0.05) \times 10^{-6}$ can be detected with an efficiency of at least $70 \%$.

The dispersing lens is optimized in the assumption of a minimum-detectable $B B R$ of $0.33 \times 10^{-7}$. The sample of 656 visible events has a minimum $B B R$ of $(0.54 \pm 0.21) \times 10^{-7}$, which is also the theoretical minimum value imposed by the holographic fiducial volume cuts. This limit is reached by the FNAL machine and HOLRED. The minimum-detected $B B R$ s for the Hawaii and HAM machines are very close to the latter value, namely $(0.56 \pm$ $0.22) \times 10^{-7}$ and $(0.60 \pm 0.23) \times 10^{-7}$, respectively. Within the large systematic errors, these values include the $B B R$ limit implied by the cuts on the holographic volume. The optical reconstruction efficiency for event vertices with a $B B R$ within the interval $0.54 \times 10^{-7}$ 
to $0.85 \times 10^{-7}$ is still close to $60 \%$ for the FNAL replay machine. The various labs were probably limited by $B B R^{\prime}$ and not $B B R$ so that they all were really close to the minimum visibility.

\section{SUMMARY}

About 220000 holograms were recorded during the 1987/88 fixed-target run at the Fermilab 15-Foot Bubble Chamber. Approximately half of these were judged to have sufficient track quality for further physics analysis. Known adverse effects which lead to the loss of events are: instabilities of the holographic recording system and the operating conditions of the bubble chamber, delays due to the changing of the operating temperature of the almost 20 tons of cryogenic liquid, unstable start-up conditions of the chamber after extended scheduled standby periods, a too early laser pulse, low $B B R$, over- and underexposure of the holograms, event vertex too close to the reference beam, and laserinduced boiling.

The two-track or optical resolution of the holographic system, i.e. the combination of the recordings system and the FNAL replay device, was visually determined at $\sim 160 \mu \mathrm{m}$ in air. In terms of the bubble chamber definition for resolution, this amounts to $\sim 80 \mu \mathrm{m}$. Bubbles with diameter of the order of $120 \mu \mathrm{m}$ were seen with good contrast and brightness.

Micro-boiling is a major source of background light. It was therefore expected to have a substantial influence on the minimum $B B R$ of the event vertices, and thus also on the holographic volume in which events were seen.

A selected sample of 3552 conventional pictures, predicted to contain at least one neutrino-induced event in the corresponding hologram within a volume of about $4.2 \mathrm{~m}^{3}$, has been examined. For $42 \%$ of the conventional pictures the predicted event was indeed found in the hologram. If the event was required to be flagged by the event trigger, then the fraction increased to $55 \%$. Approximately $12 \%$ of the events were therefore lost due to improper timing of the laser pulse. The events, consisting of bubbles with diameter of $\sim 120 \mu \mathrm{m}$ or a growth time of $1 \mathrm{~ms}$, were located within a volume of $1.4 \mathrm{~m}^{3}$.

The optical reconstruction efficiency for event vertices with $B B R$ 's larger than $(0.13 \pm 0.05) \times 10^{-6}$ was shown to exceed $70 \%$ for well-tuned holographic replay devices. Within the selected holographic fiducial volume of $4.2 \mathrm{~m}^{3}$, the lowest detected $B B R$ was $(0.54 \pm 0.21) \times 10^{-7}$, which is the lowest possible value within that volume. The optical reconstruction efficiency for that $B B R$ was $\leq 58 \%$. Of the various known sources (bubble movement, bubble growth, chamber vibrations, laser-induced boiling, and multiple reflections) which increase the minimum-detectable $B B R$, laser-induced boiling near the dispersing lens was the most likely one.

\section{Acknowledgements}

We would like to thank C. Baltay, M. Bregman, M. Hibbs, A. Schaffer, T. Woch (Columbia University), T. Hart ${ }^{\dagger}$, R. Michaels (Rutgers), E. Clayton (IC London), and S. Lomatch (IIT) for their help during the initial tests and preparation of the experiment. At Fermilab we are indebted to Prof. L. Lederman, and several groups who greatly aided us. The patient and dedicated work of the 15-Foot crew and of the operators of the EMI/IPF system is greatly appreciated. 


\section{References}

[1] D. Gabor, Nature 161 (1948) 777.

[2] D. Gabor, "Holography, 1948-1971," in: Les Prix Nobel en 1971 (Imprimerie Royal P.A. Norstedt \& Söner, Stockholm, 1972) pp. 169-201.

[3] L.O. Heflinger, R.F. Wuerker and R.E. Brooks, J. Appl. Phys. 37 (1966) 642.

[4] R.L. Powell and K.A. Stetson, J. Opt. Soc. Am. 55 (1965) 1593.

[5] B.P. Hildebrand and K.A. Haines, J. Opt. Soc. Am. 57 (1967) 155.

[6] W.T. Welford, Appl. Opt. 2 (1963) 981; J. Opt. Soc. Am. 56 (1966) 1430.

[7] R.J. Withrington, Appl. Opt. 7 (1968) 175.

[8] R.J. Withrington, "Bubble chamber holography," in: Strathclyde Conference on Application of Holography (Cambridge University Press, 1970) pp. 267-278.

[9] F.R. Eisler, Nucl. Instr. and Meth. 163 (1970) 105.

[10] M. Dykes et al., Nucl. Instr. and Meth. 179 (1981) 487.

[11] A. Hervé et al., Nucl. Instr. and Meth. 202 (1982) 417.

[12] T. Kitagaki et al., Nucl. Instr. and Meth. A 281 (1989) 81.

[13] Proposal for neutrino investigations using the hybrid neutrino spectrometer (GINES) at UNK (Protvino, 1990) (unpublished).

[14] Yu.A. Bykovskii, A.M. Zarubin and A.I. Larkin, Sov. J. Quant. Electron. 16 (1986) 1165.

[15] C. Baltay, M. Bregman, M. Hibbs, A. Schaffer, G. Harigel, F. Eisler, R. Cence, E.B. Brucker and T. Hart, "Holography in large bubble chambers: Fermilab 15Foot Bubble Chamber and CERN BEBC," Talk at BEBC Users' Meeting, CERN, November 1982.

[16] G. Harigel et al., "Holographic recording of cosmic ray tracks in BEBC," in: Photonics Applied to Nuclear Physics: Nucleophot 2, Strasbourg, 1984, eds. C. Fisher et al., CERN 85-10 (CERN, Geneva, 1985) pp. 69-71.

[17] L. Verluyten, "Holography in the FNAL 15-Foot Bubble Chamber and particle density fluctuations in neutrino interactions," Thesis, Universitaire Instelling Antwerpen, 1991.

[18] H. Bingham et al., Nucl. Instr. and Meth. A 297 (1990) 364.

[19] "15-Foot Bubble Chamber Safety Report," National Accelerator Laboratory, Batavia, Illinois, July 1972.

[20] G. Harigel and N.M. Harigel, Kerntechnik 19 (1977) 354.

[21] W.T. Welford, in: Bubble and Spark Chambers, Vol. I, ed. R.P. Shutt (Academic Press, New York, 1967) p. 234.

[22] L. Verluyten, S. Willocq, G.G. Harigel, J.K. Hawkins, W.M. Smart, W.A. Williams, M.W. Peters and H. Akbari, Nucl. Instr. and Meth. A 292 (1990) 313.

[23] L. Verluyten, S. Willocq, W.M. Smart and M.W. Peters, Nucl. Instr. and Meth. A 292 (1990) 571.

[24] M.W. Peters and R.J. Cence, in: Photonics Applied to Nuclear Physics: Nucleophot 2, Strasbourg, 1984, eds. C. Fisher et al., CERN 85-10 (CERN, Geneva, 1985) pp. 9598.

[25] M. Aderholz et al., Nucl. Instr. and Meth. A 284 (1989) 311.

[26] R. Naon, H. Bjelkhagen, R. Burnstein and L. Voyvodic, Nucl. Instr. and Meth. A 283 (1989) 24.

[27] E.B. Brucker, D. DeProspo, J. Doroshenko, P. Jacques, M. Kalelkar, E.L. Koller, M. Lauko, R. Plano and P. Stamer, "The Rutgers Holographic Analysis Machine (HAM)", Rutgers University, Internal Report, March 1988 (unpublished). 
[28] H. Akbari, "High resolution imaging of particle interactions," Ph.D. Thesis, Tufts University, June 1987 (unpublished).

[29] P.R. Nailor, "Holographic reconstruction of tracks in large volume bubble chambers," Thesis, Imperial College, London, 1989 (unpublished).

[30] G. Harigel, C. Baltay, M. Bregman, M. Hibbs, A. Schaffer, H. Bjelkhagen, J. Hawkins, W. Williams, P. Nailor, R. Michaels and H. Akbari, Appl. Opt. 25 (1986) 4102.

[31] V. Jain et al., Phys. Rev. D 41 (1990) 2057.

[32] H. Foeth, Nucl. Instr. and Meth. 176 (1980) 203.

[33] F.H. Poesposoetjipto and E. Hugentobler, Helv. Phys. Acta 43 (1970) 203.

[34] G. Horlitz, S. Wolff and G. Harigel, Nucl. Instr. and Meth. 117 (1974) 115.

[35] G. Horlitz, S. Wolff and G. Harigel, Nucl. Instr. and Meth. 115 (1974) 365.

[36] G. Harigel, P. Kunkel and M. Scheer, "Zur Blasendichte in einer PropanBlasenkammer," MPI-PA-6/63 April 1963 (unpublished);

P. Kunkel, M. Christenn and M. Scheer, Int. Colloquium on Bubble Chambers, Heidelberg, 1967, ed. H. Leutz, CERN 67-26 (CERN, Geneva, 1967) Vol. II, p. 405, and Z. Angew. Phys. 22 (1967) 410.

[37] E. Hugentobler, B. Hahn and F. Steinrisser, Helv. Phys. Acta 36 (1963) 601.

[38] W.T. Welford, Nucl. Instr. and Meth. 91 (1971) 381.

[39] K.H. Carnell and W.T. Welford, Nucl. Instr. and Meth. 92 (1971) 229. 